Pacific

Journal of

Mathematics

\title{
ONE-SIDED $M$-IDEALS AND MULTIPLIERS IN OPERATOR SPACES, I
}

David P. Blecher, Edward G. Effros, and VRej Zarikian 


\title{
ONE-SIDED $M$-IDEALS AND MULTIPLIERS IN OPERATOR SPACES, I
}

\author{
David P. Blecher, Edward G. Effros, and Vrej Zarikian
}

The theory of $M$-ideals and multiplier mappings of Banach spaces naturally generalizes to left (or right) $M$-ideals and multiplier mappings of operator spaces. These subspaces and mappings are intrinsically characterized in terms of the matrix norms. In turn this is used to prove that the algebra of left adjointable mappings of a dual operator space $X$ is a von Neumann algebra. If in addition $X$ is an operator $A$ - $B$-bimodule for $C^{*}$-algebras $A$ and $B$, then the module operations on $X$ are automatically weak* continuous. One sided $L$-projections are introduced, and analogues of various results from the classical theory are proved. An assortment of examples is considered.

\section{Introduction.}

It has long been recognized that the algebraic structure of a $C^{*}$-algebra $A$ is closely linked to its geometry as a Banach space (see [25]). This principle was illustrated in [5], and [2], p. 237, where it was shown that the closed two-sided ideals of a $C^{*}$-algebra coincide with the $M$-ideals of the underlying Banach space (see also [35]). Similarly, the center of a $C^{*}$-algebra is determined by the centralizer mappings of the Banach space [5], [9]. It was subsequently shown that these notions can be applied to a broad range of Banach space problems unrelated to operator algebra theory (see [24] for references to the extensive literature on this subject).

In this paper we show that one can similarly characterize the closed onesided ideals and one-sided multipliers in a $C^{*}$-algebra in terms of its matrix norms, i.e., its underlying operator space structure. We show that the closed one-sided ideals in a $C^{*}$-algebra are just the complete one-sided $M$-ideals (defined below) of the operator space. We also prove that the one-sided multipliers and the one-sided adjointable multipliers of an operator space (first studied independently in [10] and [39], see also [12]) have surprisingly simple matrix norm characterizations. Once again these abstract considerations have important applications elsewhere, including a striking automatic continuity result for dual modules (see Corollary 5.6). They have also led to a new characterization of the dual operator algebras [11]. 
Turning to the details, if $X$ is an operator space, a linear mapping $P$ : $X \rightarrow X$ with $P^{2}=P$ is said to be a left $M$-projection if for each $x \in X$,

$$
\|x\|=\left\|\left[\begin{array}{c}
P(x) \\
x-P(x)
\end{array}\right]\right\| .
$$

We say that $P$ is a complete left $M$-projection if for each $n \in \mathbb{N}, P_{n}$ : $M_{n}(X) \rightarrow M_{n}(X)$ is a left $M$-projection. Here $P_{n}$ is the canonical "entrywise" action of $P$ on matrices. A subspace $J$ of $X$ is a (complete) right $M$-summand if $J=P(X)$ with $P$ a (complete) left $M$-projection. Finally, a closed subspace $J$ of $X$ is a (complete) right $M$-ideal if $J^{\perp \perp}$ is a (complete) right $M$-summand. If $A$ is a unital $C^{*}$-algebra, then the complete left $M$ projections are given by $P(x)=e x$ where $e$ is an orthogonal projection in $A$. Hence the complete right $M$-summands of $A$ are the algebraic right ideals of the form $e A$. As a consequence the complete right $M$-ideals in a $C^{*}$-algebra are exactly the closed right ideals. One may similarly define the notion of a right $M$-projection by using row matrices. We have left the routine details of such reversed notions (left $M$-summands, etc.) to the reader.

As in the theory of $M$-ideals in a Banach space, it is technically useful to introduce the dual notions of one-sided L-projections, L-summands and $L$-ideals in an operator space. We also prove that complete one-sided $L$ ideals are necessarily $L$-summands, one-sided $L$-summands are Chebychev, and complete one-sided $L$ and $M$-projections are uniquely determined by their ranges. These and other "one-sided" analogues of the classical $M$ ideal theory are presented in $\S 3$. We make no attempt to be exhaustive. Additional results, together with a more detailed exposition of the basic theory may be found in [42]. We have deferred some of these topics to the sequel of this paper, and to [13].

Given an operator space $X$ and a completely isometric embedding

$$
\sigma: X \hookrightarrow B(K, H),
$$

we say that $b \in B(H)$ is a left multiplier of $X$ if $b \sigma(X) \subseteq \sigma(X)$, and we let $M_{\ell}^{\sigma}(X)$ be the algebra of all such $b \in B(H)$. To simplify the notation we will often write $X \subseteq B(K, H)$ and $b X \subseteq X$. The left multipliers in the unital $C^{*}$-algebra

$$
A_{\ell}^{\sigma}(X)=M_{\ell}^{\sigma}(X) \cap M_{\ell}^{\sigma}(X)^{*} \subseteq B(H)
$$

are said to be left adjointable. Since we have the natural inclusion map

$$
B(K, H) \hookrightarrow B(K \oplus H, K \oplus H)
$$

we may, for most purposes, restrict our attention to multipliers associated with embeddings of the form $\sigma: X \hookrightarrow B(L)$ for a Hilbert space $L$. On the other hand, we need the more general embeddings to prove the existence of Shilov embeddings (see below). 
Given an embedding (1), each $b \in M_{\ell}^{\sigma}(X)$ determines a map

$$
\varphi=L^{\sigma}(b): X \rightarrow X: x \mapsto b x,
$$

with $\|\varphi\|_{c b} \leq\|b\|$. We say that a linear map $\varphi: X \rightarrow X$ is a left multiplier map if $\varphi=L^{\sigma}(b)$ for some embedding $\sigma: X \hookrightarrow B(K, H)$ and $b \in M_{\ell}^{\sigma}(X) \subseteq$ $B(H)$. We let $M_{\ell}(X) \subseteq C B(X)$ be the set of all such maps $\varphi$. Similarly, $\varphi$ is a left adjointable multiplier map if $\varphi=L^{\sigma}(b)$ with $b \in A_{\ell}^{\sigma}(X)$, and we let $A_{\ell}(X) \subseteq C B(X)$ denote the set of all such maps $\varphi$.

Given an operator space $X$, then one can use the construction of the "noncommutative Shilov boundary" of an operator space to find an embedding $\sigma_{0}: X \hookrightarrow B(K, H)$ with the following properties:

(i) For any $\varphi \in M_{\ell}(X)$ there is a unique element $b_{0} \in M_{\ell}^{\sigma_{0}}(X)$ such that $\varphi=L^{\sigma_{0}}\left(b_{0}\right)$

(ii) for any $\varphi \in A_{\ell}(X)$ there exists a unique element $b_{0} \in A_{\ell}^{\sigma_{0}}(X)$ such that $\varphi=L^{\sigma_{0}}\left(b_{0}\right)$,

(iii) if $\varphi=L^{\sigma}\left(b_{1}\right)$ for some embedding $\sigma: X \hookrightarrow B\left(K_{1}, H_{1}\right)$ and element $b_{1} \in M_{\ell}^{\sigma}(X)$, then $\left\|b_{0}\right\| \leq\left\|b_{1}\right\|$.

(See $[\mathbf{1 0}],[\mathbf{7}, \mathbf{8}],[\mathbf{2 2}],[\mathbf{2 3}]$.) For lack of a better term, we will refer to an embedding $\sigma_{0}$ with these properties as a "Shilov embedding". The existence of such an embedding implies that $M_{\ell}(X)$ and $A_{\ell}(X)$ are subalgebras of $C B(X)$.

If $\sigma_{0}: X \hookrightarrow B(K, H)$ is a Shilov embedding, then by definition the map

$$
L^{\sigma_{0}}: M_{\ell}^{\sigma_{0}}(X) \rightarrow M_{\ell}(X) \subseteq C B(X)
$$

and its restriction

$$
A_{\ell}^{\sigma_{0}}(X) \rightarrow A_{\ell}(X) \subseteq C B(X)
$$

are algebraic isomorphisms.

Since $A_{\ell}^{\sigma_{0}}(X)$ is a $C^{*}$-algebra, it follows that the algebraic isomorphism (3) is isometric (see [36], Prop. 1.1), and we have a corresponding $C^{*}$ algebraic structure on $A_{\ell}(X)$. If $\sigma_{1}$ is another Shilov embedding, then the algebras $A_{\ell}^{\sigma_{j}}(X)(j=0,1)$ are isometrically isomorphic as unital Banach algebras. Since a unital norm-decreasing map of $C^{*}$-algebras is necessarily *-preserving (see, e.g., Lemma 5.2 below), they are isomorphic $C^{*}$-algebras, and therefore the $C^{*}$-algebraic structure on $A_{\ell}(X)$ does not depend upon the Shilov embedding. The self-adjoint projections in this $C^{*}$-algebra are the complete left $M$-projections on $X$ (see Theorem 5.1).

It is shown in [10] and [12] that although the isomorphism (2) is generally not isometric, there is a natural operator space structure on $M_{\ell}(X)$ with respect to which it is an operator algebra. In particular if $\varphi \in M_{\ell}(X)$, then the corresponding norm is given by $\|\varphi\|_{M_{\ell}(X)}=\left\|b_{0}\right\|$, where $\varphi=L^{\sigma_{0}}\left(b_{0}\right)$ for an arbitrary Shilov embedding $\sigma_{0}$ and $b_{0} \in M_{\ell}^{\sigma_{0}}(X)$. 
One of the main objectives of this paper is find intrinsic characterizations of the left multiplier and left adjointable multiplier maps. In order to state these criteria, we need some definitions. An element $a$ of a unital Banach algebra $A$ is said to be hermitian if $\left\|e^{i t a}\right\|=1$ for all $t \in \mathbb{R}$ (see [14]). If $X$ is an operator space, we say that a mapping $\varphi: X \rightarrow X$ is completely hermitian if it is a hermitian element of $C B(X)$, or equivalently if, for each $n \in \mathbb{N}$, the map $\varphi_{n}: M_{n}(X) \rightarrow M_{n}(X)$ is hermitian in $B\left(M_{n}(X)\right)$.

We let the space $C_{2}(X)=M_{2,1}(X)$ of $2 \times 1$ column matrices over an operator space $X$ have its canonical operator space structure. Given a linear mapping $\varphi: X \rightarrow X$, we define the column mapping $\tau_{\varphi}^{c}: C_{2}(X) \rightarrow C_{2}(X)$ by

$$
\tau_{\varphi}^{c}\left(\left[\begin{array}{l}
x \\
y
\end{array}\right]\right)=\left[\begin{array}{c}
\varphi(x) \\
y
\end{array}\right]
$$

Theorem 1.1. Suppose that $X$ is an operator space, and that $\varphi: X \rightarrow X$ is a linear mapping. Then the following are equivalent:

(a) There exists a completely isometric embedding $X \hookrightarrow B(H)$ such that $\varphi(x)=b x$ for some $b \in B(H)$ with $\|b\| \leq 1$ (respectively, $b=b^{*}, b$ an orthogonal projection);

(b) $\tau_{\varphi}^{c}$ is completely contractive (respectively, $\tau_{\varphi}^{c}$ is completely hermitian, $\varphi$ is a complete left $M$-projection).

From our previous discussion of multipliers we may use a Shilov embedding in (a). It follows that the first statement in (a) is equivalent to the condition that $\varphi \in M_{\ell}(X)$ and $\|\varphi\|_{M_{\ell}(X)} \leq 1$. We will use this result in $\S 5$ to prove that the left adjointable multiplier algebra $A_{\ell}(X)$ of a dual operator space $X$ (i.e., $X$ is the dual of an operator space) is a von Neumann algebra. A consequence of this is that $C^{*}$-algebraic operator bimodule operations on a dual operator space are automatically weak* continuous. We also consider some functorial properties of the multiplier mappings.

In $\S 6$ we give various examples. In particular we prove that the complete right $M$-ideals in a Hilbert $C^{*}$-module are exactly the closed submodules, and we list some consequences of this. We also observe that the classical $M$ ideals of Banach spaces, and the "complete $M$-ideals" of the second author and Ruan, may be viewed as particular examples of complete left $M$-ideals.

The theory of one-sided ideals and multipliers in a unital $C^{*}$-algebra $A$ has a long history. It was shown in $[\mathbf{1 6}]$ and $[\mathbf{3 0}]$ that they are in one-toone correspondence with the closed faces of the state space $S(A)$. These faces are particularly well-behaved, and a corresponding theory of "split faces" of a convex set was studied in $[4,3]$. This theory played a key role in the Alfsen-Schultz characterization of the state spaces of $C^{*}$-algebras (see $[6])$. On the other hand, K. H. Werner considered a related notion for operator systems (these are matrix ordered spaces), and he defined a notion of multipliers of such spaces [38], [41]. E. Kirchberg considered 
multipliers of a certain class of operator spaces in [26]. Arveson was the first to consider "Shilov representations" $[\mathbf{7}, \mathbf{8}]$, of operator spaces, and this theory was further developed by Hamana. See the remarks Added in Proof on page 316 for additional background material.

\section{Some operator space preliminaries.}

We refer the reader to the book [21] as a general reference to the theory of operator spaces, and for help with any of the details below.

An operator space $X$ is a vector space together with distinguished norms on each matrix space $M_{n}(X)$ which are linked by the relations

$$
\begin{aligned}
\|x \oplus y\| & =\max \{\|x\|,\|y\|\}, \\
\|\alpha x \beta\| & \leq\|\alpha\|\|x\|\|\beta\| .
\end{aligned}
$$

Here $\alpha, \beta$ are scalar matrices, and the $\oplus$ refers to the "diagonal direct sum" of matrices (see [33]). These "square matrix" norms uniquely determine norms on each "rectangular matrix" space $M_{m, n}(X)$. By considering matrices over the latter space, we see that $M_{m, n}(X)$ is again an operator space. We let

$$
C_{n}(X)=M_{n, 1}(X), R_{n}(X)=M_{1, n}(X),
$$

with these operator space structures, and in particular, we let $C_{n}=C_{n}(\mathbb{C})$ and $R_{n}=R_{n}(\mathbb{C})$. We have the natural complete isometries

$$
\begin{aligned}
& C_{n}(X)=C_{n} \check{\otimes} X=C_{n} \otimes_{h} X, \\
& R_{n}(X)=R_{n} \check{\otimes} X=X \otimes_{h} R_{n},
\end{aligned}
$$

where $\check{\otimes}$ and $\otimes_{h}$ denote the usual spatial and Haagerup tensor products for operator spaces (see e.g., Chapters 7-9 in [21]). On the other hand, we let

$$
\begin{gathered}
C_{n}[X]=C_{n} \hat{\otimes} X=X \otimes_{h} C_{n} \\
R_{n}[X]=R_{n} \hat{\otimes} X=R_{n} \otimes_{h} X,
\end{gathered}
$$

where $\hat{\otimes}$ denotes the projective operator space tensor product. We have the identifications

$$
\begin{aligned}
\left(C_{n}(X)\right)^{*} & =R_{n}\left[X^{*}\right], \quad\left(R_{n}(X)\right)^{*}=C_{n}\left[X^{*}\right], \\
\left(C_{n}[X]\right)^{*} & =R_{n}\left(X^{*}\right), \quad\left(R_{n}[X]\right)^{*}=C_{n}\left(X^{*}\right),
\end{aligned}
$$

where in each case we use the pairings

$$
\left\langle\left[\begin{array}{l}
x_{1} \\
x_{2} \\
\vdots \\
x_{n}
\end{array}\right],\left[\begin{array}{lll}
f_{1} & f_{2} \ldots f_{n}
\end{array}\right]\right\rangle=\sum_{k} f_{k}\left(x_{k}\right)=\left\langle\left[x_{1} x_{2} \ldots x_{n}\right],\left[\begin{array}{l}
f_{1} \\
f_{2} \\
\vdots \\
f_{n}
\end{array}\right]\right\rangle .
$$

An essential distinction between $C_{2}(X)$ and $C_{2}[X]$ can be seen from the following lemma. It should be noted that the obvious modification of this result is true for rows and columns of arbitrary length. 
Lemma 2.1. Suppose that $X$ is an operator space and that $x, y \in X$. Then

$$
\left\|\left[\begin{array}{l}
x \\
y
\end{array}\right]\right\|_{C_{2}(X)} \leq\left(\|x\|^{2}+\|y\|^{2}\right)^{1 / 2}
$$

and

$$
\left\|\left[\begin{array}{l}
x \\
y
\end{array}\right]\right\|_{C_{2}[X]} \geq\left(\|x\|^{2}+\|y\|^{2}\right)^{1 / 2} .
$$

Proof. We may assume that $X$ is a subspace of $B(H)$ for some Hilbert space $H$. Then

$$
\left\|\left[\begin{array}{l}
x \\
y
\end{array}\right]\right\|_{C_{2}(X)}^{2}=\left\|\left[\begin{array}{ll}
x^{*} & y^{*}
\end{array}\right]\left[\begin{array}{l}
x \\
y
\end{array}\right]\right\|=\left\|x^{*} x+y^{*} y\right\| \leq\|x\|^{2}+\|y\|^{2} .
$$

Equivalently, if we let $X \oplus_{2} X$ denote the vector space $X \oplus X$ with the norm

$$
\|(x, y)\|=\left(\|x\|^{2}+\|y\|^{2}\right)^{1 / 2}
$$

then the mapping

$$
\theta_{X}^{c}: X \oplus_{2} X \rightarrow C_{2}(X):(x, y) \mapsto\left[\begin{array}{l}
x \\
y
\end{array}\right]
$$

is a contraction. Of course the same applies to the corresponding mapping $\theta_{X}^{r}: X \oplus_{2} X \rightarrow R_{2}(X)$. If we define

$$
\eta_{X}^{c}: C_{2}[X] \rightarrow X \oplus_{2} X:\left[\begin{array}{l}
x \\
y
\end{array}\right] \mapsto(x, y),
$$

then it is evident that $\left(\eta_{X}^{c}\right)^{*}=\theta_{X^{*}}^{r}$, and since $\theta_{X^{*}}^{r}$ is contractive, that is also true for $\eta_{X}^{c}$, i.e., we have (5).

It is immediate from the axioms for an operator space that

$$
\|x\| \leq\left\|\left[\begin{array}{l}
x \\
y
\end{array}\right]\right\|_{C_{2}(X)},
$$

and from (5) that

$$
\|x\| \leq\left\|\left[\begin{array}{l}
x \\
y
\end{array}\right]\right\|_{C_{2}[X]} .
$$

We will need the following result in Proposition 6.10.

Lemma 2.2. Let $X$ be an operator space. Then the map $R_{2}\left(R_{2}[X]\right) \rightarrow$ $R_{2}\left[R_{2}(X)\right]$ defined by

$$
\left[\begin{array}{llll}
u & v & w & x
\end{array}\right] \mapsto\left[\begin{array}{llll}
u & w & v & x
\end{array}\right]
$$

is a complete isometry. 
Proof. We have from above the natural complete isometries

$$
\begin{aligned}
R_{2} \check{\otimes}\left(R_{2} \hat{\otimes} X\right) & \cong\left(R_{2} \hat{\otimes} X\right) \check{\otimes} R_{2} \\
& \cong\left(R_{2} \otimes_{h} X\right) \otimes_{h} R_{2} \\
& \cong R_{2} \otimes_{h}\left(X \otimes_{h} R_{2}\right) \\
& \cong R_{2} \hat{\otimes}\left(X \check{\otimes} R_{2}\right) \\
& \cong R_{2} \hat{\otimes}\left(R_{2} \check{\otimes} X\right) .
\end{aligned}
$$

If we successively apply these identifications to an elementary tensor on the left, we obtain

$$
\begin{aligned}
{\left[\begin{array}{ll}
\alpha & \beta
\end{array}\right] \otimes\left(\left[\begin{array}{ll}
\gamma & \delta
\end{array}\right] \otimes x\right) } & \mapsto\left(\left[\begin{array}{ll}
\gamma & \delta
\end{array}\right] \otimes x\right) \otimes\left[\begin{array}{ll}
\alpha & \beta
\end{array}\right] \\
& \mapsto\left[\begin{array}{ll}
\gamma & \delta
\end{array}\right] \otimes\left(x \otimes\left[\begin{array}{ll}
\alpha & \beta
\end{array}\right]\right) \\
& \mapsto\left[\begin{array}{ll}
\gamma & \delta
\end{array}\right] \otimes\left(\left[\begin{array}{ll}
\alpha & \beta
\end{array}\right] \otimes x\right),
\end{aligned}
$$

i.e.,

$$
\left[\begin{array}{llll}
(\alpha \gamma) x & (\alpha \delta) x & (\beta \gamma) x & (\beta \delta) x
\end{array}\right] \mapsto\left[\begin{array}{llll}
(\gamma \alpha) x & (\gamma \beta) x & (\delta \alpha) x & (\delta \beta) x
\end{array}\right]
$$

which coincides with (8). This extends by linearity to arbitrary tensors on the left.

\section{One-sided $M$-projections and $L$-projections.}

If $X$ is a vector space, we say that a linear mapping $P: X \rightarrow X$ is a projection if $P^{2}=P$ (for Hilbert space operators we will also insist that the mapping be self-adjoint). If $I$ is the identity mapping, it follows that $I-P$ is also a projection. If $P$ is a projection, then the linear mappings

$$
\begin{aligned}
& \nu_{P}^{c}: \quad X \rightarrow C_{2}(X): x \mapsto\left[\begin{array}{c}
P(x) \\
x-P(x)
\end{array}\right], \\
& \mu_{P}^{c} \quad: \quad C_{2}(X) \rightarrow X:\left[\begin{array}{l}
x \\
y
\end{array}\right] \mapsto P(x)+y-P(y),
\end{aligned}
$$

satisfy $\mu_{P}^{c} \circ \nu_{P}^{c}=I$. We have corresponding mappings $\nu_{P}^{r}: X \rightarrow R_{2}(X)$ and $\mu_{P}^{r}: R_{2}(X) \rightarrow X$ which satisfy $\mu_{P}^{r} \circ \nu_{P}^{r}=I$.

We recall that if $X$ is a Banach space, then a projection $P: X \rightarrow X$ is an $M$-projection if for every $x \in X$ we have

$$
\|x\|=\max \{\|P(x)\|,\|x-P(x)\|\} .
$$

If $X$ is an operator space, we say that $P$ is a complete $M$-projection if for each $n \in \mathbb{N}, P_{n}: M_{n}(X) \rightarrow M_{n}(X)$ is an $M$-projection. It is known that $M$-projections need not be complete $M$-projections (see [20]).

From the introduction, $P: X \rightarrow X$ is a left $M$-projection if and only if

$$
\nu_{P}^{c}: X \rightarrow C_{2}(X): x \mapsto\left[\begin{array}{c}
P(x) \\
x-P(x)
\end{array}\right]
$$


is an isometric injection. Using simple matrix manipulations it is evident that $P$ is a complete left $M$-projection if and only if $\nu_{P}^{c}$ is completely isometric. Owing to the fact that

$$
\left[\begin{array}{l}
b \\
a
\end{array}\right]=\left[\begin{array}{ll}
0 & 1 \\
1 & 0
\end{array}\right]\left[\begin{array}{l}
a \\
b
\end{array}\right]
$$

it is evident that if $P$ is a (complete) left $M$-projection, then the same is true for $I-P$.

If $e$ is a (self-adjoint) projection in a unital $C^{*}$-algebra $A$, then $P(x)=e x$ is a left $M$-projection on $A$ since

$$
\begin{aligned}
\left\|\left[\begin{array}{c}
P(x) \\
x-P(x)
\end{array}\right]\right\|^{2} & =\left\|\left[\begin{array}{c}
e x \\
x-e x
\end{array}\right]\right\|^{2}=\left\|\left[\begin{array}{ll}
x^{*} e & x^{*}-x^{*} e
\end{array}\right]\left[\begin{array}{c}
e x \\
x-e x
\end{array}\right]\right\| \\
& =\left\|x^{*} e x+x^{*}(1-e) x\right\|=\left\|x^{*} x\right\|=\|x\|^{2} .
\end{aligned}
$$

If $x \in M_{n}(A)$, then $P_{n}(x)=e_{n} x$ where $e_{n}=e \oplus \cdots \oplus e$ is a projection in $M_{n}(A)$, and it follows that $P$ is complete left $M$-projection.

Lemma 3.1. Suppose that $X$ is an operator space. A projection $P: X \rightarrow X$ is both a complete left and a complete right $M$-projection if and only if it is a complete $M$-projection.

Proof. If $P$ is a complete left and right $M$-projection, then $\|x\|=\left\|\nu_{P}^{c}(x)\right\|=$ $\left\|\left(\nu_{P}^{r}\right)_{2,1}\left(\nu_{P}^{c}(x)\right)\right\|$, and thus

$$
\begin{aligned}
\|x\| & =\left\|\left[\begin{array}{c}
P(x) \\
x-P(x)
\end{array}\right]\right\|=\left\|\left[\begin{array}{cc}
P^{2}(x) & (I-P) P(x) \\
P(I-P)(x) & (I-P)^{2}(x)
\end{array}\right]\right\| \\
& =\left\|\left[\begin{array}{cc}
P(x) & 0 \\
0 & x-P(x)
\end{array}\right]\right\|=\max \{\|P(x)\|,\|x-P(x)\|\} .
\end{aligned}
$$

This applies as well to matrices. Conversely if $P$ is a complete $M$-projection, then the mapping

$$
\theta: X \rightarrow M_{2}(X): x \mapsto(P x) \oplus(x-P x)
$$

is completely isometric. It follows that

$$
\begin{aligned}
\left\|\left[\begin{array}{c}
P(x) \\
x-P(x)
\end{array}\right]\right\| & =\left\|\theta_{2,1}\left(\left[\begin{array}{c}
P(x) \\
x-P(x)
\end{array}\right]\right)\right\| \\
& =\max \{\|P x\|,\|x-P x\|\}=\|x\| .
\end{aligned}
$$

Again it is easy to generalize this to matrices. A similar argument may be applied to row matrices.

The following result will be useful in our discussion of duality.

Proposition 3.2. If $X$ is an operator space and $P: X \rightarrow X$ is a projection, then $P$ is a complete left $M$-projection if and only if $\mu_{P}^{c}$ and $\nu_{P}^{c}$ are both completely contractive. 
Proof. If $\nu_{P}^{c}$ is completely isometric, then

$$
\|P(x)+y-P(y)\|=\left\|\left[\begin{array}{c}
P(x) \\
y-P(y)
\end{array}\right]\right\| \leq\left\|\left[\begin{array}{c}
P(x) \\
x-P(x) \\
P(y) \\
y-P(y)
\end{array}\right]\right\|=\left\|\left[\begin{array}{l}
x \\
y
\end{array}\right]\right\|,
$$

and thus $\mu_{P}^{c}$ is contractive. These calculations work as well for matrices. The converse is trivial since if two complete contractions compose to the identity, then the first is completely isometric.

As in the Banach space theory, left $M$-projections have certain automatic continuity properties.

Proposition 3.3. Suppose that $X$ is an operator space which is also a dual Banach space. Then any left $M$-projection $P: X \rightarrow X$ is weak ${ }^{*}$ continuous.

Proof. A standard argument in functional analysis shows that it suffices to prove that the unit balls of $P(X)$ and $(I-P)(X)$ are weak* closed. By symmetry of $P$ and $I-P$ it is enough to prove the former. Let us suppose that $\left\{y_{\nu}\right\}$ is a net in $P(X)$ with $\left\|y_{\nu}\right\| \leq 1$, converging weak* to an element $x \in X$. If we let $y=P(x)$ and $z=(I-P)(x)$, it follows that $y_{\nu}^{\prime}=y_{\nu}-y$ converges weak* to $z$. Scaling by $\frac{1}{2}$, we may suppose that we have a net $\left\|y_{\nu}\right\| \leq 1$, converging weak* to a $z \in(I-P)(X)$. For any $t>0$, we have $y_{\nu}+t z \rightarrow(1+t) z$. Hence using the fact that norm closed balls in $X$ are weak* closed, and (4), we see that

$$
(1+t)^{2}\|z\|^{2} \leq \sup _{\nu}\left\|y_{\nu}+t z\right\|^{2}=\sup _{\nu}\left\|\left[\begin{array}{c}
y_{\nu} \\
t z
\end{array}\right]\right\|_{C_{2}(X)}^{2} \leq 1+t^{2}\|z\|^{2} .
$$

Letting $t \rightarrow \infty$ shows that $z=0$.

We say that a projection $P: X \rightarrow X$ is a left $L$-projection if

$$
\|x\|=\left\|\left[\begin{array}{c}
P(x) \\
x-P(x)
\end{array}\right]\right\|_{C_{2}[X]},
$$

or equivalently if $\nu_{P}^{c}: X \rightarrow C_{2}[X]$ is isometric. We say that $P$ is a complete left $L$-projection if the mapping $\nu_{P}^{c}: X \rightarrow C_{2}[X]$ is a complete isometry.

Proposition 3.4. If $X$ is an operator space and $P: X \rightarrow X$ is a projection, then $P$ is a complete left $L$-projection if and only if $\nu_{P}^{c}: X \rightarrow C_{2}[X]$ and $\mu_{P}^{c}: C_{2}[X] \rightarrow X$ are completely contractive.

Proof. As in the proof of Proposition 3.2, the key point is to show that if $P$ is a complete left $L$-projection, i.e., $\nu_{P}^{c}$ is completely isometric, then $\mu_{P}^{c}$ is a 
complete contraction. The truncation mapping

$$
\rho: C_{4} \rightarrow C_{2}:\left[\begin{array}{l}
\alpha \\
\beta \\
\gamma \\
\delta
\end{array}\right] \mapsto\left[\begin{array}{l}
\alpha \\
\delta
\end{array}\right]
$$

is completely contractive and thus, by the "functoriality" of the projective tensor product, it induces a complete contraction $\rho \otimes$ id : $C_{4}[X] \rightarrow C_{2}[X]$. We have a commutative diagram

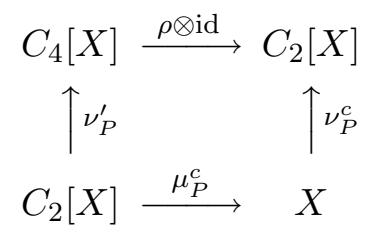

where $\nu_{P}^{\prime}=\mathrm{id}_{C_{2}} \otimes \nu_{P}^{c}$. This is because for any $x, y \in X$,

$$
\begin{aligned}
(\rho \otimes \mathrm{id}) \circ \nu_{P}^{\prime}\left[\begin{array}{l}
x \\
y
\end{array}\right] & =(\rho \otimes \mathrm{id})\left[\begin{array}{l}
P x \\
x-P x \\
P y \\
y-P y
\end{array}\right] \\
& =\left[\begin{array}{l}
P x \\
y-P y
\end{array}\right]=\nu_{P}^{c}(P x+y-P y) .
\end{aligned}
$$

It follows that $(\rho \otimes \mathrm{id}) \circ \nu_{P}^{\prime}$ has range in $\nu_{P}^{c}(X)$. By hypothesis,

$$
\nu_{P}^{c}: X \rightarrow \nu_{P}^{c}(X)
$$

is a complete isometry, and thus

$$
\mu_{P}^{c}=\left(\nu_{P}^{c}\right)^{-1} \circ(\rho \otimes \mathrm{id}) \circ \nu_{P}^{\prime}
$$

is a complete contraction.

The proofs of Proposition 3.2 and Proposition 3.4 do not generalize to left $M$ - and left $L$-projections. For this reason it might be useful to consider a related notion. We say that a projection $P: X \rightarrow X$ is a strong left $M$-projection if $\nu_{P}^{c}: X \rightarrow C_{2}(X)$ and $\mu_{P}^{c}: C_{2}(X) \rightarrow X$ are contractive, and we similarly define strong left $L$-projections. The reader will see that the duality relationships considered below are also valid for these "strong" one-sided projections. In fact most of the results of this section which are stated for "complete one-sided projections and summands and ideals", are also valid with "complete" replaced by "strong".

Corollary 3.5. If $X$ is an operator space and $P: X \rightarrow X$ is a projection, then $P$ is a complete left $M$-projection if and only if $P^{*}$ is a complete right $L$-projection. Similarly $P$ is a complete right $L$-projection if and only if $P^{*}$ is a complete left $M$-projection. 
Proof. For any $x \in X$ and $f, g \in X^{*}$,

$$
\begin{aligned}
\left\langle\nu_{P}^{c}(x),\left[\begin{array}{ll}
f & g
\end{array}\right]\right\rangle & =\left\langle\left[\begin{array}{c}
P(x) \\
x-P(x)
\end{array}\right],\left[\begin{array}{ll}
f & g
\end{array}\right]\right\rangle \\
& =\langle P(x), f\rangle+\langle x-P(x), g\rangle \\
& =\left\langle x, P^{*}(f)+g-P^{*}(g)\right\rangle \\
& =\left\langle x, \mu_{P^{*}}^{r}([f g])\right\rangle,
\end{aligned}
$$

and thus $\left(\nu_{P}^{c}\right)^{*}=\mu_{P^{*}}^{r}$. Similarly, $\left(\mu_{P}^{c}\right)^{*}=\nu_{P^{*}}^{r}$. It follows from Proposition 3.2 and Proposition 3.4, and basic operator space duality, that $P$ is a complete left $M$-projection if and only if $P^{*}$ is a complete right $L$-projection, and similarly $P$ is a complete right $L$-projection if and only if $P^{*}$ is a complete left $M$-projection.

We recall from the introduction that a subspace $J$ of an operator space $X$ is a (complete) right $M$-summand of $X$ if it is the range of a (complete) left $M$-projection. We say that $J$ is a (complete) right $L$-summand if it is the range of a (complete) left $L$-projection. We note that if $P: X \rightarrow X$ is a bounded projection, then the same is true for $P^{*}$, and we have

$$
P(X)^{\perp}=\operatorname{ker} P^{*}=\left(I-P^{*}\right)\left(X^{*}\right) .
$$

We thus have:

Corollary 3.6. If $X$ is an operator space and $J \subseteq X$ is a complete right $M$-summand, then $J^{\perp}$ is a complete left $L$-summand, and if $J \subseteq X$ is a complete right L-summand, then $J^{\perp}$ is a complete left $M$-summand.

A subspace $J$ of a Banach space $X$ is said to be proximinal (respectively, Chebychev) if for each $x \in X$, the set

$$
\mathcal{P}_{J}(x)=\{h \in J:\|x-h\|=\|x-J\|\}
$$

is nonempty (respectively, has one point). If $P: X \rightarrow X$ is a left $M$ projection, $J=P(X)$, and $x \in X$, then

$$
P(x) \in \mathcal{P}_{J}(x),
$$

since if $x \in X$ and $h \in J$, then

$$
\|x-h\|=\left\|\left[\begin{array}{c}
P(x-h) \\
(I-P)(x-h)
\end{array}\right]\right\|=\left\|\left[\begin{array}{l}
P(x)-h \\
x-P(x)
\end{array}\right]\right\| \geq\|x-P(x)\| .
$$

It follows that right $M$-summands are proximinal. A similar argument with (7) shows that right $L$-summands are also proximinal.

Proposition 3.7. If $P$ is a left L-projection with $J=P(X)$, then $\mathcal{P}_{J}(x)=$ $\{P(x)\}$, and thus $J$ is Chebychev. 
Proof. If $h \in J$, then from (5),

$$
\begin{aligned}
\|x-h\|^{2} & =\left\|\left[\begin{array}{c}
P(x-h) \\
(I-P)(x-h)
\end{array}\right]\right\|_{C_{2}[X]}^{2} \\
& =\left\|\left[\begin{array}{c}
P(x)-h \\
x-P(x)
\end{array}\right]\right\|_{C_{2}[X]}^{2} \\
& \geq\|P(x)-h\|^{2}+\|x-P(x)\|^{2} .
\end{aligned}
$$

It follows that if $h \in \mathcal{P}_{J}(x)$, then $\|x-h\|=\|x-P(x)\|$ and $h=P(x)$.

Corollary 3.8. If $J$ is a complete right $M$-summand (respectively, right $L$-summand), then there is only one complete left $M$-projection (respectively, left $L$-projection) with range $J$.

Proof. Given left $L$-projections $P$ and $Q$ with $J=P(X)=Q(X)$, we have

$$
\{P(x)\}=\mathcal{P}_{J}(x)=\{Q(x)\}
$$

for $x \in X$, and therefore $P=Q$. If $P$ and $Q$ are complete left $M$-projections with $J=P(X)=Q(X)$, then

$$
\operatorname{ker} P^{*}=J^{\perp}=\operatorname{ker} Q^{*}
$$

implies that the right $L$-projections $I-P^{*}$ and $I-Q^{*}$ have the same range. Thus $I-P^{*}=I-Q^{*}$ and $P=Q$.

In the introduction we defined a subspace $J$ of an operator space $X$ to be a right $M$-ideal if $J^{\perp \perp}$ is a right $M$-summand. From the next result we see that it is equivalent to assume that $J^{\perp}$ is a left $L$-summand. As in the Banach space theory, this next result also shows that there is no need to define $L$-ideals, since they must coincide with $L$-summands.

Proposition 3.9. If $J$ is a closed subspace of an operator space $X$ for which $J^{\perp}$ is a complete right $M$-summand, then $J$ is a complete left $L$-summand. Indeed any complete right $M$-summand in a dual operator space $X^{*}$ is the annihilator of a complete left L-summand in $X$.

Proof. Let us suppose that $J^{\perp}$ is a complete right $M$-summand in $X^{*}$ and let $P$ be the complete left $M$-projection onto $J^{\perp}$. From Proposition 3.3, $P$ is weak* continuous. It follows that $P=Q^{*}$ for a projection $Q: X \rightarrow X$. That implies that

$$
(I-Q)(X)^{\perp}=\operatorname{ker}(I-P)=P\left(X^{*}\right)=J^{\perp}
$$

and thus $J=(I-Q)(X)$. Since $Q^{*}$ is a complete left $M$-projection, $Q$ and $I-Q$ are complete right $L$-projections. The proof for the second assertion is similar. 
In fact stronger versions of the last few results are true. We omit the proofs, which are very simple and identical to their classical versions (see [24]):

Theorem 3.10. In the following, $X$ is an operator space.

(a) Suppose that $P$ is a complete left $M$-projection on $X$. If $Q$ is a contractive projection on $X$ with $\operatorname{Ran} Q=\operatorname{Ran} P$, then $P=Q$.

(b) Suppose that $P$ is a complete right $L$-projection on $X$. If $Q$ is a contractive projection on $X$ with $\operatorname{ker} Q=\operatorname{ker} P$, then $Q=P$.

(c) If there exists a contractive projection from $X$ onto a complete right $M$-ideal $J$ of $X$, then $J$ is a complete right $M$-summand. Moreover such a contractive projection is then unique.

(d) If $J$ is a complete right $M$-ideal in $X$, and if $J$ is a dual Banach space, then $J$ is a complete right $M$-summand in $X$.

(e) If $X$ is a dual operator space, and if $J$ is a weak*-closed complete right $M$-ideal of $X$, then $J$ is a complete right $M$-summand in $X$ which is the annihilator of a complete left $L$-summand in $X_{*}$.

The "complete" hypothesis in the results above may be weakened to the "strong" condition briefly alluded to earlier. In light of the topics to be discussed in $\S 6.5$ below, (e) may be regarded as an operator space generalization of the result that weak*-closed submodules of self-dual $C^{*}$-modules are orthogonally complemented. (d) is related to the well-known fact that if a closed submodule of a Hilbert $C^{*}$-module is self-dual, then it is orthogonally complemented.

Proposition 3.11. Suppose that $X$ and $Y$ are operator spaces. If $P$ is a complete left $M$-projection on $X$, then

$$
P \otimes \text { id }: X \check{\otimes} Y \rightarrow X \check{\otimes} Y
$$

is a complete left $M$-projection. If $P$ is a complete left $L$-projection on $X$, then

$$
P \otimes \text { id }: X \hat{\otimes} Y \rightarrow X \hat{\otimes} Y
$$

is a complete left L-projection.

Proof. Owing to the functorial properties of the tensor product, and using Proposition 3.2, the mappings $\mu_{P}^{c}$ and $\nu_{P}^{c}$ tensor with id ${ }_{Y}$ to give complete contractions

$$
X \check{\otimes} Y \rightarrow C_{2}(X) \check{\otimes} Y \rightarrow X \check{\otimes} Y .
$$

The first relation then follows again from Proposition 3.2, together with the simple identification

$$
C_{2}(X \check{\otimes} Y)=C_{2}(X) \check{\otimes} Y .
$$

The second relation follows similarly. 


\section{Multipliers.}

In order to illustrate the definition of the multiplier mappings, let us consider an elementary proof for the characterization of complete left $M$-projections given in Theorem 1.1.

Proposition 4.1. The complete left $M$-projections in an operator space $X$ are just the mappings $P(x)=$ ex for a completely isometric embedding $X \hookrightarrow$ $B(H)$ and an orthogonal projection $e \in B(H)$.

Proof. If $P: X \rightarrow X$ is a complete left $M$-projection, then let us fix an embedding $X \subseteq B(H)$. By definition, the mapping

$$
\sigma: X \hookrightarrow B(H \oplus H): x \mapsto\left[\begin{array}{cc}
P(x) & 0 \\
(I-P)(x) & 0
\end{array}\right]
$$

is completely isometric. We have that

$$
\sigma(P(x))=\left[\begin{array}{cc}
P(x) & 0 \\
0 & 0
\end{array}\right]=\left[\begin{array}{ll}
1 & 0 \\
0 & 0
\end{array}\right] \sigma(x),
$$

and thus $e=\left[\begin{array}{ll}1 & 0 \\ 0 & 0\end{array}\right] \in B(H \oplus H)$ is the desired left projection relative to the embedding $\sigma$. The converse is immediate (see the calculation before Lemma 3.1).

We will give some other characterizations of the complete left $M$-projections in Theorem 5.1.

In order to prove the remaining parts of Theorem 1.1, it is useful to consider a bimodule version of Hamana's theory of injective envelopes [12]. Given unital $C^{*}$-algebras $A$ and $B$, an operator space $X$ which is also a left $A$-module is called a left operator $A$-module if $\|a x\| \leq\|a\|\|x\|$ for all matrices $a \in M_{n}(A)$ and $x \in M_{n}(X)$. We assume that the module action is unitary, i.e., that $1 x=x$ for all $x$. There is a similar definition for right operator $B$-modules, and for operator $A-B$-modules. Bimodule mappings are defined in the usual manner.

We say that an operator $A-B$-bimodule $Z$ is an injective bimodule if given an inclusion of $A-B$-bimodules $X \subseteq Y$, any completely contractive $A$-B-bimodule mapping $\theta: X \rightarrow Z$ extends to an $A$-B-bimodule mapping $Y \rightarrow Z$. An inclusion of $A$-B-bimodules $X \subseteq Y$ is rigid if given a completely contractive $A$-B-bimodule mapping $\varphi: Y \rightarrow Y$ such that $\varphi_{\mid X}=\operatorname{id}_{X}$, it follows that $\varphi=\operatorname{id}_{Y}$. We say that an injective operator $A-B$-bimodule $Z$ is an operator $A-B$-bimodule injective envelope of an operator $A$ - $B$-bimodule $X$, if there exists a completely isometric rigid $A$-B-bimodule inclusion $X \hookrightarrow$ $Z$. Following Hamana's argument $[\mathbf{2 2}, \mathbf{2 3}]$, one can see that the $A-B$ bimodule injective envelope is unique in the obvious sense. If $A=B=\mathbb{C}$, then we are simply talking about the injective envelope $I(X)$ of an operator 
space $X$, as discussed in $[\mathbf{2 2}, \mathbf{2 3}, \mathbf{3 4}]$. The following result was proved in [12], Corollary 2.6. In fact we only need the $\mathbb{C} \oplus \mathbb{C}-\mathbb{C}$-module version of this result, which may be proved by elementary methods.

Lemma 4.2. An operator $A-B$-bimodule $Y$ is injective as an operator $A-$ $B$-bimodule if and only if it is injective as an operator space. The injective envelope $I(X)$ of the operator space $X$ may be regarded as the operator $A$ $B$-bimodule injective envelope of $X$.

We will be considering infinite matrices over operator spaces. Given an operator space $X$ and cardinals $m, n$, we have a corresponding operator space $M_{m, n}(X)$ of all matrices for which the finite truncations are uniformly bounded (see [21]). If $\varphi: X \rightarrow Y$ is a completely bounded mapping of operator spaces, the mapping

$$
\varphi_{m, n}: M_{m, n}(X) \rightarrow M_{m, n}(Y):\left[x_{i j}\right] \rightarrow\left[\varphi\left(x_{i j}\right)\right]
$$

satisfies $\left\|\varphi_{m, n}\right\|_{c b}=\|\varphi\|_{c b}$. If we let $D_{n}$ denote the diagonal matrices in $M_{n}$ it is evident that $M_{m, n}(X)$ is an operator $D_{m}-D_{n^{-}}$-bimodule. The $D_{m}-D_{n^{-}}$ bimodule mappings

$$
\varphi: M_{m, n}(X) \rightarrow M_{m, n}(Y),
$$

are just those for which there exist linear mappings $\varphi_{i j}: X \rightarrow Y$ with

$$
\varphi\left(\left[x_{i j}\right]\right)=\left[\varphi_{i j}\left(x_{i j}\right)\right] .
$$

We will only need the following result for $m=2, n=1$, in which case there is also an elementary direct proof. We have included the general case since it is of independent interest.

Lemma 4.3. For any cardinals $m, n$, we have a natural identification

$$
M_{m, n}(I(X)) \cong I\left(M_{m, n}(X)\right),
$$

i.e., $M_{m, n}(I(X))$ is an injective envelope of $M_{m, n}(X)$.

Proof. From the previous lemma it suffices to prove that $M_{m, n}(I(X))$ is the $D_{m}-D_{n}$-bimodule injective envelope of $M_{m, n}(X)$. To see this we first note that if $Z$ is injective, then so is $M_{m, n}(Z)$. This follows since if $\pi: B(H) \rightarrow Z$ is a surjective completely contractive projection, then

$$
\pi_{m, n}: M_{m, n}(B(H)) \rightarrow M_{m, n}(Z)
$$

is a completely contractive projection of the injective operator space $M_{m, n}(B(H)) \cong B\left(H^{n}, H^{m}\right)$ onto $M_{m, n}(Z)$. If $\varphi: M_{m, n}(I(X)) \rightarrow$ $M_{m, n}(I(X))$ is a $D_{m}-D_{n}$-bimodule complete contraction such that $\varphi_{\mid M_{m, n}(X)}$ $=\operatorname{id}_{M_{m, n}(X)}$, then in particular, $\varphi_{i j}(x)=x$ for $x \in X$, and therefore $\varphi_{i j}(x)=x$ for $x \in I(X)$. It follows that $\varphi=\mathrm{id}$ and we see that

$$
M_{m, n}(X) \subseteq M_{m, n}(I(X))
$$


is a rigid bimodule inclusion. Thus $M_{m, n}(I(X))$ is a bimodule injective envelope of $M_{m, n}(X)$ and from Lemma 4.2 it is an operator space injective envelope of $M_{m, n}(X)$.

Lemma 4.4. Suppose that $X$ is the second dual of a ternary system. Then for some cardinal $J, M_{J}(X)$ is completely isometric to a von Neumann algebra.

This result is in the folklore of the Morita equivalence theory of von Neumann algebras. It may be found in [10] Lemma 5.8, and a more general result assuming that $X$ is a weakly closed injective ternary system may also be deduced from results in $[\mathbf{1 7}]$.

We will use the following simple but elegant result of R.R. Smith (see [13]). We include a sketch of the proof for the sake of completeness.

Lemma 4.5 ([13]). Suppose that $M$ is a von Neumann algebra. Then a mapping $\varphi: M \rightarrow M$ has the form $\varphi(x)=b x$ for some $b \in M$ with $\|b\| \leq 1$ if and only if the column mapping

$$
\tau_{\varphi}^{c}: C_{2}(M) \rightarrow C_{2}(M):\left[\begin{array}{l}
x \\
y
\end{array}\right] \mapsto\left[\begin{array}{c}
\varphi(x) \\
y
\end{array}\right]
$$

is contractive.

Proof. For the difficult direction, we suppose that $\tau_{\varphi}^{c}$ is contractive, and apply $\tau_{\varphi}^{c}$ to the column in $C_{2}(M)$ with entries $e$ and $1-e$, for an orthogonal projection $e \in M$. We obtain $\varphi(e)^{*} \varphi(e)+(1-e) \leq 1$ and thus

$$
(1-e) \varphi(e)^{*} \varphi(e)(1-e)=0,
$$

giving $\varphi(e)(1-e)=0$. But this relation also holds for the projection $1-e$, i.e., we have $\varphi(1-e) e=0$. We conclude that

$$
\varphi(e)=\varphi(e) e=\varphi(1) e .
$$

Since the linear span of the projections is norm dense in $M, \varphi(x)=b x$ for all $x \in M$, where $b=\varphi(1)$.

Theorem 4.6. Suppose that $X$ is an operator space and that $\varphi: X \rightarrow X$ is a linear mapping. Then there exists a completely isometric embedding $X \hookrightarrow B(H)$ and an operator $b \in B(H)_{1}$ with $\varphi(x)=b x$ for all $x \in X$ if and only if

$$
\tau_{\varphi}^{c}: C_{2}(X) \rightarrow C_{2}(X):\left[\begin{array}{l}
x \\
y
\end{array}\right] \mapsto\left[\begin{array}{c}
\varphi(x) \\
y
\end{array}\right]
$$

is completely contractive. 
Proof. Let us suppose that $\tau_{\varphi}^{c}$ is completely contractive. From Lemma 4.3 and Lemma $4.2, C_{2}(I(X))=I\left(C_{2}(X)\right)$ is the $D_{2}-D_{1}$-bimodule injective envelope of $C_{2}(X)$. Thus we may extend the $D_{2}-D_{1}$-bimodule mapping

$$
\tau_{\varphi}^{c}: C_{2}(X) \rightarrow C_{2}(X)
$$

to a bimodule mapping

$$
\theta: C_{2}(I(X)) \rightarrow C_{2}(I(X)):\left[\begin{array}{l}
x \\
y
\end{array}\right] \mapsto\left[\begin{array}{l}
\theta_{1}(x) \\
\theta_{2}(y)
\end{array}\right] .
$$

Since $\theta_{2}$ restricts to the identity on $X$ and $X \subseteq I(X)$ is rigid, $\theta_{2}=\mathrm{id} I(X)$. Thus if we let $\widetilde{\varphi}=\theta_{1}: I(X) \rightarrow I(X)$, it follows that

$$
\tau_{\widetilde{\varphi}}^{c}: C_{2}(I(X)) \rightarrow C_{2}(I(X)):\left[\begin{array}{l}
x \\
y
\end{array}\right] \mapsto\left[\begin{array}{c}
\widetilde{\varphi}(x) \\
y
\end{array}\right]
$$

is completely contractive.

If we use the natural identification

$$
C_{2}\left(I(X)^{* *}\right)=C_{2}(I(X))^{* *},
$$

it follows that

$$
\tau_{\widetilde{\varphi}^{* *}}^{c}=\left(\tau_{\widetilde{\varphi}}^{c}\right)^{* *}: C_{2}\left(I(X)^{* *}\right) \rightarrow C_{2}\left(I(X)^{* *}\right):\left[\begin{array}{l}
x \\
y
\end{array}\right] \mapsto\left[\begin{array}{c}
\widetilde{\varphi}^{* *}(x) \\
y
\end{array}\right]
$$

is completely contractive. We have by $[\mathbf{2 3}, \mathbf{3 4}]$ that $I(X)$ is completely isometric to a ternary system $e A(1-e)$, where $A$ is a $C^{*}$-algebra and $e$ is an orthogonal projection in $A$. It follows that $I(X)^{* *}$ is completely isometric to the weakly closed ternary system $e A^{* *}(1-e)$, and from Lemma 4.4 there is a cardinal $J$ such that $R=M_{J}\left(I(X)^{* *}\right)$ is a von Neumann algebra. The corresponding mapping

$$
\bar{\varphi}=\left(\widetilde{\varphi}^{* *}\right)_{J}: M_{J}\left(I(X)^{* *}\right) \rightarrow M_{J}\left(I(X)^{* *}\right)
$$

extends the mapping

$$
\varphi_{J}: M_{J}(X) \rightarrow M_{J}(X)
$$

and from the identification

$$
C_{2}\left(M_{J}\left(I(X)^{* *}\right)\right)=M_{J}\left(C_{2}\left(I(X)^{* *}\right)\right)
$$

we have that

$$
\tau_{\bar{\varphi}}^{c}: C_{2}(R) \rightarrow C_{2}(R):\left[\begin{array}{l}
x \\
y
\end{array}\right] \mapsto\left[\begin{array}{c}
\bar{\varphi}(x) \\
y
\end{array}\right]
$$

is completely contractive.

From Lemma 4.5, we have that there is a contraction $b \in R$ such that $\bar{\varphi}(x)=b x$ for all $x \in R$. Let us fix an index $j_{0} \in J$, and if $x \in X$, define

$$
[x]_{j_{0}} \in M_{J}(X) \subseteq M_{J}\left(I(X)^{* *}\right)
$$


to be the matrix with $x$ at the $j_{0}, j_{0}$ entry and zero elsewhere. Then

$$
[\varphi(x)]_{j_{0}}=\varphi_{J}\left([x]_{j_{0}}\right)=\bar{\varphi}\left([x]_{j_{0}}\right)=b[x]_{j_{0}} .
$$

The last product here needs a word of clarification. The point is that $[x]_{j_{0}}$ is in $M_{J}\left(I(X)^{* *}\right)$ which is only linearly completely isometric, via a mapping $\rho$ say, to the von Neumann algebra $R$. Then the statement above reads, more precisely,

$$
\rho\left([\varphi(x)]_{j_{0}}\right)=b \rho\left([x]_{j_{0}}\right) .
$$

Defining an embedding of $X$ in $R$ by $\sigma_{1}(x)=\rho\left([x]_{j_{0}}\right)$, we see that $\varphi$ is a left multiplier mapping.

We leave the simple argument for the converse to the reader.

We remark that the mapping $\sigma_{1}$ constructed in the previous proof cannot take the place of the Shilov embedding $\sigma_{0}$ described in the introduction, since in particular the corresponding mapping $L: M_{\ell}^{\sigma_{1}}(X) \rightarrow C B(X)$ is not one-to-one. On the other hand with a little effort, and using results in [12], it may be seen that a compression of $\sigma_{1}$ has the desired properties of $\sigma_{0}$. The space of relative multipliers with respect to this compression will then coincide with the $I M_{\ell}(X)$ formulation of the left multiplier algebra given in $[12]$.

The procedure used in the last proof of passing from $X$ to $I(X)$ to $I(X)^{* *}$ and finally to the von Neumann algebra $R \cong M_{J}\left(I(X)^{* *}\right)$ was first used in $[10] \S 5$. These steps provide a useful and essentially canonical technique for embedding an arbitrary operator space $X$ into a von Neumann algebra.

Corollary 4.7. If $\varphi$ is a linear mapping on a right $C^{*}$-module, then $\varphi$ is a contractive module mapping if and only if $\tau_{\varphi}^{c}$ is completely contractive.

Proof. This follows from the last theorem and the fact from [10] A.4 that $M_{\ell}(Z)$ for a $C^{*}$-module $Z$ is the set of bounded module mappings on $Z$.

Corollary 4.8. Suppose that $X$ is an operator space and that $\varphi: X \rightarrow X$ is a linear mapping. Then $\varphi(x)=u x$ for a unitary $u \in A_{\ell}(X)$ if and only if $\tau_{\varphi}^{c}$ is a completely isometric bijection.

Proof. One direction is clear. For the other, let us use a Shilov embedding $\sigma_{0}: X \hookrightarrow B(K, H)$ (see $\S 1$ ). Then applying Theorem 4.6 to $\varphi$ and $\varphi^{-1}$, we obtain contractions $b, c \in B(H)$ with $b c x=x=c b x$. Since $L^{\sigma_{0}}: M_{\ell}^{\sigma_{0}}(X) \rightarrow$ $C B(X)$ is one-to-one, it follows that $b c=c b=1$, and thus $b=c^{-1}$. Since $b$ and $c$ are both contractions, $b$ is unitary.

We can now prove the remaining assertion in Theorem 1.1, namely the characterization of left self-adjointable multipliers.

Theorem 4.9. If $X$ is an operator space, then a mapping $\varphi: X \rightarrow X$ is a left self-adjoint multiplier if and only if $\tau_{\varphi}^{c}$ is completely hermitian. 
Proof. One direction is fairly clear. For the other, we have that

$$
\begin{aligned}
\exp i t \tau_{\varphi}^{c}\left(\left[\begin{array}{l}
x \\
y
\end{array}\right]\right) & =\left(I+i t \tau_{\varphi}^{c}+\frac{\left(i t \tau_{\varphi}^{c}\right)^{2}}{2 !}+\ldots\right)\left(\left[\begin{array}{l}
x \\
y
\end{array}\right]\right) \\
& =\left[\begin{array}{c}
\exp i t \varphi(x) \\
e^{i t} y
\end{array}\right] \\
& =\left[\begin{array}{cc}
1 & 0 \\
0 & e^{i t}
\end{array}\right]\left[\begin{array}{c}
\exp i t \varphi(x) \\
y
\end{array}\right]
\end{aligned}
$$

and thus

$$
\exp i t \tau_{\varphi}^{c}=\left[\begin{array}{cc}
1 & 0 \\
0 & e^{i t}
\end{array}\right] \tau_{\exp i t \varphi}^{c}
$$

If $\tau_{\varphi}^{c}$ is completely hermitian, then $\exp i t \tau_{\varphi}^{c}$ is a completely isometric surjection, and that is also the case for $\tau_{\exp i t \varphi}^{c}$. From Corollary 4.8, $\psi(t)=\exp i t \varphi$ is a unitary element of $A_{\ell}(X)$. Since $t \mapsto \psi(t)$ is a norm continuous oneparameter group of unitaries in the $C^{*}$-algebra $A_{\ell}(X)$, it follows that $\varphi$ is a self-adjoint element in $A_{\ell}(X)$.

\section{Some applications.}

If $x, y \in B(H)$ then we say that $x \perp y$ if $x^{*} y=0$. Similarly for subsets $E, F \subseteq B(H)$, we write $E \perp F$ if $x^{*} y=0$ for all $x \in E, y \in F$.

Theorem 5.1. If $P$ is a projection on an operator space $X$, then the following are equivalent (and are also equivalent to the conditions in Proposition 4.1):

(a) $P$ is a complete left $M$-projection.

(b) $\tau_{P}^{c}$ is completely contractive.

(c) $P$ is an orthogonal projection in the $C^{*}$-algebra $A_{\ell}(X)$.

(d) $P \in M_{\ell}(X)$ with multiplier norm $\leq 1$.

(e) There exists an embedding $\sigma: X \hookrightarrow B(H)$ such that

$$
\sigma(P(X)) \perp \sigma((I-P)(X)) .
$$

In (e), $\sigma$ may be taken to be a Shilov embedding.

Proof. That (b) is equivalent to (d), and that (a) implies (d) follows from Theorem 4.6 and Proposition 4.1. Let us assume (d). If we use a Shilov embedding $\sigma_{0}: X \hookrightarrow B(K, H)$, it follows that $P=L^{\sigma_{0}}(b)$, where $b \in B(H)$ is a contraction. Since $L^{\sigma_{0}}$ is one-to-one, $b^{2}=b$, and from elementary operator theory, $b=b^{*}$ is an orthogonal projection on $H$. Thus $P=L^{\sigma_{0}}(b)$ is an orthogonal projection in $A_{\ell}(X)$ and we have (c). Given (c), it is immediate that $P$ is the image of an orthogonal projection in $A_{\ell}^{\sigma_{0}}(X)$. From Proposition 4.1 that implies (a).

Given (a), there exists by Proposition 4.1 an embedding $\sigma: X \hookrightarrow B(H)$, and an orthogonal projection $e \in B(H)$ with $\sigma(P x)=e \sigma(x)$ for all $x \in$ 
$X$. From the above discussion we see that we can take $\sigma$ to be a Shilov embedding and it is evident that (e) holds for this $\sigma$. Finally, given (e), we will show that $P$ is adjointable in the sense of $\S 4$ of [10]. If $x, y \in X$, then

$$
\sigma(P x)^{*} \sigma(y)=\sigma(P x)^{*} \sigma(P y+(I-P) y)=\sigma(P x)^{*} \sigma(P y),
$$

and also

$$
\sigma(x)^{*} \sigma(P y)=\left(\sigma(P y)^{*} \sigma(x)\right)^{*}=\left(\sigma(P y)^{*} \sigma(P x)\right)^{*}=\sigma(P x)^{*} \sigma(P y) .
$$

Since these are equal, $P$ is adjointable. It follows from [10] that $P$ satisfies (c).

We now wish to investigate the $C^{*}$-algebra $A_{\ell}(X)$ in the case that $X$ is the operator space dual of an operator space.

The following is well-known (see Lemma A.4.2 in [21] and [14], I.10.10).

Lemma 5.2. Given an operator $d$ on a Hilbert space $H$ with $\|d\| \leq 1$, we have that $d=d^{*}$ if and only if $\|1+i t d\| \leq \sqrt{1+t^{2}}$ for all $t \in \mathbb{R}$. If $d$ is an element of a unital Banach algebra $A$ such that $\|1+i t d\| \leq \sqrt{1+t^{2}}$ for all $t \in \mathbb{R}$, then it is hermitian in $A$.

Lemma 5.3. Given an operator space $X$ and a left multiplier $\varphi: X \rightarrow X$ such that $\|\varphi\|_{M_{\ell}(X)} \leq 1$, it follows that $\tau_{\varphi}^{c}$ is a left multiplier of $C_{2}(X)$ with $\left\|\tau_{\varphi}^{c}\right\|_{M_{\ell}\left(C_{2}(X)\right)} \leq 1$. If $\varphi$ is a self-adjoint or adjointable left multiplier, then the same is true for $\tau_{\varphi}^{c}$.

Proof. Let us suppose that $X \subseteq B\left(H_{0}\right)$ is a Shilov embedding and that $\varphi(x)=b x$, where $b \in B\left(H_{0}\right)$. We have a natural embedding $\sigma: C_{2}(X) \hookrightarrow$ $B\left(H_{0}^{2}\right)$ defined by

$$
\sigma\left(\left[\begin{array}{l}
x \\
y
\end{array}\right]\right)=\left[\begin{array}{ll}
x & 0 \\
y & 0
\end{array}\right]
$$

and we have that

$$
\sigma\left(\tau_{\varphi}^{c}\left(\left[\begin{array}{l}
x \\
y
\end{array}\right]\right)\right)=\left[\begin{array}{cc}
b x & 0 \\
y & 0
\end{array}\right]=\left[\begin{array}{ll}
b & 0 \\
0 & I
\end{array}\right] \sigma\left(\left[\begin{array}{l}
x \\
y
\end{array}\right]\right)
$$

where $b \oplus I$ is a contractive left multiplier of $\sigma\left(C_{2}(X)\right)$. If $b$ is self-adjoint, then that is also the case for $b \oplus I$, and if the real and imaginary parts of $b$ are left multipliers, that is also the case for $b \oplus I$, hence the remaining assertions are evident.

Theorem 5.4. If $X$ is the operator space dual of an operator space, then $A_{\ell}(X)$ is a von Neumann algebra.

Proof. Let us suppose that $X$ is the dual of the operator space $X_{*}$. We have that $A_{\ell}(X)$ is a Banach subalgebra of $C B(X)$. On the other hand, we may identify $C B(X)$ with the operator space dual $\left(X \hat{\otimes} X_{*}\right)^{*}$. To show that $A_{\ell}(X)$ is a dual Banach space it suffices to prove that it is closed in the 
weak* topology in $C B(X)$, and for that it suffices to prove that its unit ball $D=A_{\ell}(X)_{1}$ is weak* closed in the unit ball $C B(X)_{1}$. Since $X \hat{\otimes} X_{*}$ is the norm completion of $X \otimes X_{*}$, the latter determines the same topology on $C B(X)_{1}$, and thus given $\varphi_{\nu}, \varphi \in C B(X)_{1}, \varphi_{\nu} \rightarrow \varphi$ in the weak ${ }^{*}$ topology if and only if $\varphi_{\nu}(x) \rightarrow \varphi(x)$ in the weak ${ }^{*}$ topology for each $x \in X$.

Suppose that $\varphi_{\nu} \in D_{s a}, \varphi \in C B(X)_{1}$ and that $\varphi_{\nu}(x) \rightarrow \varphi(x)$ in the weak* topology for each $x \in X$. If we use the duality

$$
\left(R_{2}\left[X_{*}\right]\right)^{*}=C_{2}(X)
$$

it is evident that

$$
\tau_{\varphi_{\nu}}^{c}\left(\left[\begin{array}{l}
x \\
y
\end{array}\right]\right)=\left[\begin{array}{c}
\varphi_{\nu}(x) \\
y
\end{array}\right] \rightarrow\left[\begin{array}{c}
\varphi(x) \\
y
\end{array}\right]
$$

in the weak* topology for any $x, y \in X$. Hence $\tau_{\varphi_{\nu}}^{c} \rightarrow \tau_{\varphi}^{c}$ in the weak*topology of $C B\left(C_{2}(X)\right) \cong\left(C_{2}(X) \hat{\otimes} R_{2}\left[X_{*}\right]\right)^{*}$. This follows by considerations similar to those mentioned at the end of the last paragraph, but with $X$ replaced by $C_{2}(X)$. From Lemma 5.2 and Lemma 5.3, $\left\|1+i t \tau_{\varphi_{\nu}}^{c}\right\|_{c b} \leq$ $\sqrt{1+t^{2}}$ for all $t \in \mathbb{R}$, and since norm closed balls are weak ${ }^{*}$-closed, $\| 1+$ $i t \tau_{\varphi}^{c} \|_{c b} \leq \sqrt{1+t^{2}}$ for all $t \in \mathbb{R}$. From Lemma 5.2, $\tau_{\varphi}^{c}$ is a hermitian element of the Banach algebra $C B\left(C_{2}(X)\right)$, and we have from Theorem 4.9 that $\varphi \in A_{\ell}(X)_{s a}$. On the other hand, since the norm closed unit balls in $C B(X)$ are weak* closed, $\varphi \in D_{s a}$. We conclude that $D_{s a}$ and $A_{\ell}(X)_{s a}$ are weak* closed.

Finally, let us suppose that $\varphi_{\nu} \in D, \varphi \in C B(X)_{1}$, and that $\varphi_{\nu} \rightarrow \varphi$ in the weak* topology. Since $C B(X)_{1}$ is compact in the weak* topology, by passing to a subnet twice we may assume that

$$
\operatorname{Re} \varphi_{\nu}=(1 / 2)\left(\varphi_{\nu}+\varphi_{\nu}^{*}\right) \rightarrow \psi_{1}
$$

and

$$
\operatorname{Im} \varphi_{\nu}=(1 / 2 i)\left(\varphi_{\nu}-\varphi_{\nu}^{*}\right) \rightarrow \psi_{2}
$$

in the weak* topology (we are using the involution in $A_{\ell}(X)$ ). It follows that $\varphi=\psi_{1}+i \psi_{2}$, and from the previous argument, $\psi_{i} \in A_{\ell}(X)_{s a}$. As in the self-adjoint case we have that $\|\varphi\| \leq 1$ hence $D$ and therefore $A_{\ell}(X)$ are weak* closed.

This result is an important tool in our theory, since it allows the introduction of von Neumann algebra methods to the study of dual operator spaces. For example, we see immediately that a dual operator space $X$ has no nontrivial complete left $M$-projections if and only if $A_{\ell}(X)=\mathbb{C}$. In general, the set of complete left $M$-projections on a dual operator space $X$ is a complete lattice; and there is a spectral theorem for left adjointable operators on $X$. We plan to discuss more such consequences in the sequel to this paper. 
Theorem 5.5. If $X$ is a dual operator space then any $\varphi \in A_{\ell}(X)$ is weak*continuous.

Proof. It suffices to prove that $\varphi$ is weak* continuous on the unit ball of $X$. Since $A_{\ell}(X)$ is a von Neumann algebra, $\varphi$ is a norm limit of a sequence $\varphi_{n}$, where each $\varphi_{n}$ is a linear combination of projections. The restrictions of these mappings to the unit ball of $X$ converge uniformly. From Theorem 5.1 the projections in $A_{\ell}(X)$ are the $M$-projections on $X$, and from Proposition 3.3 they are weak* continuous. It follows that each $\varphi_{n}$ is weak* continuous, and since a uniform limit of weak* continuous functions is weak* continuous, we conclude that $\varphi$ is weak ${ }^{*}$ continuous on the unit ball of $X$.

We note that we can also prove the above corollary by using the fact that any element $a \in A_{\ell}(X)$ with $0 \leq a \leq 1$ is a corner of a projection in $M_{2}\left(A_{\ell}(X)\right)=A_{\ell}\left(M_{2}(X)\right)$.

Corollary 5.6. If $X$ is a dual operator space and it is an operator $A-B$ bimodule for $C^{*}$-algebras $A$ and $B$, then the mapping $x \mapsto$ axb for $a \in A$ and $b \in B$ is automatically weak* continuous on $X$.

Proof. From [15] Corollary 3.2, there exists a completely isometric embedding $\Theta: X \hookrightarrow B(H)$ and $*$-representations $\pi_{1}$ and $\pi_{2}$ of $A$ and $B$ respectively on $H$ such that $\Theta(a x b)=\pi_{1}(a) \Theta(x) \pi_{2}(b)$ for $a \in A, b \in B$ and $x \in X$. Changing notation, let us assume that $X, A, B \subseteq B(H)$. Since the mapping $x \mapsto a x$ is in $A_{\ell}(X)$, we have from Theorem 5.5 that it is weak* continuous. On the other hand since $y \mapsto y b$ is in $A_{r}(X)$, it is also weak* continuous. It follows that

$$
x \mapsto a x b=(a x) b
$$

is weak* continuous.

Corollary 5.7. Any dual operator space $X$ is a normal dual $A_{\ell}(X)-A_{r}(X)$ bimodule in the sense of [18], i.e., the trilinear mapping $A_{\ell}(X) \times X \times$ $A_{r}(X) \rightarrow X$ is weak ${ }^{*}$-continuous in each variable.

Proof. Suppose that $a_{i} \in A_{\ell}(X)$ is a net converging weak* to $a$, and that $x \in X$. Then since the weak ${ }^{*}$-topology on $A_{\ell}(X)$ is inherited from $C B(X)=$ $\left(X \hat{\otimes} X_{*}\right)^{*}$, we have that $\psi\left(a_{i}(x b)\right) \rightarrow \psi(a(x b))$ for $\psi \in X_{*}$. The same argument applies to the third variable, and continuity in $x$ follows from the previous corollary.

Some further applications of these results to operator modules are given in $[\mathbf{1 1}]$.

We may also use Theorem 1.1 to study functorial properties of left multiplier mappings. Given a subspace $Y$ of an operator space $X$ and a left multiplier mapping $\varphi: X \rightarrow X$ such that $\varphi(Y) \subseteq Y$, it is trivial that the 
restriction $\varphi^{\prime}=\varphi_{\mid Y}$ is a left multiplier of $Y$. The following is perhaps less evident.

Proposition 5.8. Suppose that $Y$ is a closed subspace of an operator space $X$, and that $\varphi \in M_{\ell}(X)_{1}$ is such that $\varphi(Y) \subseteq Y$. Then the induced quotient mapping $\varphi^{\prime \prime}: X / Y \rightarrow X / Y$ is an element of $M_{\ell}(X / Y)_{1}$. If in addition $\varphi \in A_{\ell}(X)$, and $\varphi^{*}(Y) \subseteq Y$, then $\varphi^{\prime} \in A_{\ell}(Y)$ and $\varphi^{\prime \prime} \in A_{\ell}(X / Y)$.

Proof. Let us suppose that $\left\|\tau_{\varphi}^{c}\right\|_{c b} \leq 1$. From the definition of the quotient operator space structure (applied to rectangular matrices) we have the identification

$$
C_{2}(X / Y)=C_{2}(X) / C_{2}(Y) .
$$

Thus an element $\left[\begin{array}{l}\bar{x} \\ \bar{y}\end{array}\right] \in C_{2}(X / Y)$, with norm less than 1 is the quotient image of an element $\left[\begin{array}{l}x \\ y\end{array}\right] \in C_{2}(X)$ with norm less than 1 . We have that $\left[\begin{array}{c}\varphi^{\prime \prime}(\bar{x}) \\ \bar{y}\end{array}\right]$ is the quotient image of $\left[\begin{array}{c}\varphi(x) \\ y\end{array}\right]$, and thus

$$
\left\|\left[\begin{array}{c}
\varphi^{\prime \prime}(\bar{x}) \\
\bar{y}
\end{array}\right]\right\| \leq\left\|\left[\begin{array}{c}
\varphi(x) \\
y
\end{array}\right]\right\|<1,
$$

from which it follows that $\left\|\tau_{\varphi^{\prime \prime}}^{c}\right\| \leq 1$. A similar argument can be used on matrices.

Let $B$ denote the $\varphi \in A_{\ell}(X)$ such that $\varphi(Y) \subseteq Y$ and $\varphi^{*}(Y) \subseteq Y$. Then $B$ is a *-subalgebra of $A_{\ell}(X)$, and we have from above that $\varphi \mapsto \varphi^{\prime \prime}$ is a norm decreasing unital homomorphism from $B$ into $M_{\ell}(X / Y)$. It is evident from Lemma 5.2 that the image of a self-adjoint element of $B$ is again self-adjoint, and thus this mapping sends $B=B_{s a}+i B_{s a}$ into $A_{\ell}(X / Y)$.

\section{Examples.}

6.1. It was shown in $\S 4.22$ of $[\mathbf{1 0}]$ that if $X$ is a Banach space, then $A_{\ell}(\mathrm{MIN}(X))$ coincides with the classical centralizer algebra $Z(X)$ of $X$. Since the projections of $Z(X)$ are the $M$-projections, whereas the projections in $A_{\ell}(\operatorname{MIN}(X))$ are the complete left $M$-projections on the operator space $\operatorname{MIN}(X)$, these mappings coincide. It follows that the complete right $M$-summands and complete right $M$-ideals of $\operatorname{MIN}(X)$ are the $M$-summands and $M$-ideals of $X$. Since in general $\operatorname{MAX}(X)^{*}=\operatorname{MIN}\left(X^{*}\right)$, we also see that complete left $L$-projections (respectively, complete right $L$-summands) in $\operatorname{MAX}(X)$ are the $L$-projections (respectively, $L$-summands) in $X$.

6.2. From Lemma 3.1, the "complete $M$-projections" considered in [20] are just the complete left $M$-projections which are also complete right $M$ projections. Hence it follows from 3.10 (a) that the "complete $M$-summands" 
coincide with the complete left $M$-summands which are also complete right $M$-summands. In turn, the "complete $M$-ideals" of [20] are the complete left $M$-ideals which are also complete right $M$-ideals. There is an operator space version of the centralizer algebra of a Banach space which is appropriate to this "complete two-sided" theory, which we will consider elsewhere. One description of this algebra is the left adjointable multipliers which are also right adjointable.

As we indicated in the introduction, the complete right $M$-ideals in a $C^{*}$-algebra coincide with the closed right ideals, and the complete right $M$ summands are the "principal right ideals" of the form $e A$ for an orthogonal projection $e \in M(A)$, the multiplier algebra of $A$. (Indeed in [13] we show that the word "complete" is unnecessary here.) We consider two generalizations of this observation.

6.3. If $A$ is an operator algebra, we let $L M(A)$ be the left multiplier algebra of $A$ (this is equal to $A$ if $A$ is unital).

Proposition 6.4. If $A$ is a (possibly non-self-adjoint) operator algebra with contractive approximate identity, then the complete right $M$-summands of $A$ are exactly the principal right ideals $e A$ for an orthogonal projection $e \in$ $L M(A)$. The complete right $M$-ideals of $A$ are exactly the closed right ideals of $A$ which possess a left contractive approximate identity.

Proof. The first assertion is a consequence of 4.17 in [10], which states that $M_{\ell}(A)=L M(A)$ (this fact may also be proved more directly). Hence the complete left $M$-projections on $A$ are exactly the orthogonal projections $e \in \operatorname{LM}(A)$. If $A$ is unital, this part of the argument would be easier.

It is well-known that $A^{* *}$ is an operator algebra with the Arens product. If $J$ is a complete right $M$-ideal of $A$, then $J^{* *}=J^{\perp \perp}=\bar{J}^{w *}$ is, by the first part, equal to a principal right ideal $e A^{* *}$. Here $e \in A^{* *}$ is an orthogonal projection. Considered as subsets of $A^{* *}$, we have $J A \subset J^{* *}$. But also $J A \subset A$. So $J A \subset J^{* *} \cap A=J$ by basic functional analysis. So $J$ is a right ideal of $A$. Since $A^{* *}$ is unital, $e \in J^{* *}$, and $e$ is a left identity for $J^{* *}$. There exists a net in Ball $(J)$ which converges to $e$ in the weak* topology. By a well-known argument using the fact that the weak closure of a convex set equals its norm closure, one may replace the above net with a left contractive approximate identity for $J$ (see e.g., Theorem 2.2 in [19] for details).

Conversely, if $J$ is a closed right ideal of $A$ with a contractive left approximate identity, then $J^{* *}$ is a subalgebra of $A^{* *}$ with a left identity $e$ of norm 1 by [14] 28.7. Note that $e$ is an orthogonal projection in $A^{* *}$. Moreover $J^{* *} A^{* *} \subset J^{* *}$ by a routine argument approximating elements in $X^{* *}$ by weak*-converging nets of elements in $X$ (see e.g., [19] Theorem 2.2). We have

$$
J^{* *}=e J^{* *} \subset e A^{* *} \subset J^{* *} .
$$


Thus $J^{* *}=e A^{* *}$ is a complete right $M$-summand of $A^{* *}$ by the first part, so that $J$ is a complete right $M$-ideal of $A$.

There is a stronger result due to Zarikian [42] which is valid, in which the hypothesis "complete" is weakened.

6.5. We next consider the one-sided $M$-structure of Hilbert $C^{*}$-modules.

Theorem 6.6. The complete right $M$-ideals in a right Hilbert $C^{*}$-module are exactly the closed right submodules. The complete right $M$-summands are the orthogonally complemented right submodules.

Proof. The last statement is clear from Theorem 5.1, since for any right Hilbert $C^{*}$-module $X$, we have that $A_{\ell}(X)$ is the algebra of adjointable operators on $X$. Thus the complete left $M$-projections are exactly the adjointable projections on $X$.

We may assume by Cohen's factorization theorem that the right Hilbert $C^{*}$-module $X$ is full over a $C^{*}$-algebra $D$. We refer to [28] for information on self-dual $W^{*}$-modules. We will also use the following facts mentioned at the end of $\S 5$ in $[\mathbf{1 0}]$. We believe that these facts are essentially folklore. Namely, the second dual of the linking $C^{*}$-algebra for $X$, is the "linking $W^{*}$-algebra" for $X^{* *}$, and the last space $X^{* *}$ is a self-dual right $C^{*}$-module over $D^{* *}$. As is often very helpful in $C^{*}$-module theory, one may view the computations below as taking place within these linking algebras.

If $Y$ is a complete right $M$-ideal of a full right Hilbert $C^{*}$-module $X$ over $D$, then $Y^{\perp \perp}=\bar{Y}^{w *}=Y^{* *}$ is a complete right $M$-summand of $X^{* *}$. But by the above, the complete left $M$-projection on $X^{* *}$ corresponding to $Y^{\perp \perp}$ is a $D^{* *}$-module map. Thus $Y^{\perp \perp}=Y^{* *}$ is a $D^{* *}$-submodule of $X^{* *}$. Hence viewed as subsets of $X^{* *}$, we have that $Y D \subset Y^{\perp \perp} \cap X$. But the latter space is just $Y$, by basic functional analysis. Thus $Y$ is a $D$-submodule of $X$.

Conversely, if $Y$ is a $D$-submodule of a full Hilbert $C^{*}$-module $X$ over $D$, then $Y^{\perp \perp}=\bar{Y}^{w *}=Y^{* *}$ is a weak*-closed $D^{* *}$-submodule of $X^{* *}$. This may be seen from a routine argument approximating elements in $X^{* *}$ by weak*-converging nets of elements in $X$.

It is a well-known fact that a weak ${ }^{*}$-closed submodule of a self-dual $W^{*}$ module is orthogonally complemented. Since we are not aware of a precise reference in the literature for this we give a short proof: Suppose that $Z$ is a weak* closed submodule of a self-dual right $C^{*}$-module $X$ over a von Neumann algebra $M$. Suppose that $N$ is the von Neumann algebra acting on the left of $X$ (which may be viewed as the set of bounded adjointable $M$-module maps on $X$ ), and let $\mathcal{I}$ be the weak ${ }^{*}$-closure of $Z \bar{X}$ in $N$. Here $Z \bar{X}$ is the span of the rank one operators $z \otimes x$ for $z \in Z, x \in X$. Let $\mathcal{L}$ be the linking $W^{*}$-algebra of $X$, and consider the subspace of $\mathcal{L}$ which has $\mathcal{I}$ and $Z$ as its first row, and zero entries on the second. This subspace is 
a weak* closed right ideal in $\mathcal{L}$, and therefore equals $E \mathcal{L}$ for a projection $E \in \mathcal{L}$. It is easy to check that $E$ has only one nonzero entry, namely its 1-1-entry, and this is the desired projection in $N$ onto $Z$.

Since $X^{* *}$ is a self-dual $W^{*}$-module it follows that $Y^{\perp \perp}$ is complemented, i.e., there exists an adjointable projection on $X^{* *}$ with range $Y^{\perp \perp}$. So by the first part, $Y^{\perp \perp}$ is a complete right $M$-summand, so that $Y$ is a complete right $M$-ideal.

The following apparently new result follows from this and Theorem 3.10 (it can also be proved directly).

Corollary 6.7. There is at most one contractive linear projection from a Hilbert $C^{*}$-module onto a closed submodule. If there exists such a projection then it is an adjointable module map, so that the $C^{*}$-submodule is complemented orthogonally in the sense of Hilbert $C^{*}$-module theory.

6.8. We may also describe the one-sided $M$-structure of various Hilbertian operator spaces. In this discussion we let $H$ denote a Hilbert space, and $H_{c}$, $H_{r}$ and $H_{0}$ denote the column, row, and Pisier's self-dual quantizations of $H$ (see, e.g., $[\mathbf{2 1}])$.

Lemma 6.9. Let $\xi, \eta \in H$. Then

$$
\begin{aligned}
\left\|\left[\begin{array}{c}
\xi \\
\eta
\end{array}\right]\right\|_{C_{2}\left(H_{c}\right)} & =\left\|\left[\begin{array}{l}
\xi \\
\eta
\end{array}\right]\right\|_{C_{2}\left[H_{c}\right]}=\left\|\left[\begin{array}{ll}
\xi & \eta
\end{array}\right]\right\|_{R_{2}\left(H_{r}\right)} \\
& =\left\|\left[\begin{array}{ll}
\xi & \eta
\end{array}\right]\right\|_{R_{2}\left[H_{r}\right]}=\sqrt{\|\xi\|^{2}+\|\eta\|^{2}} .
\end{aligned}
$$

This also coincides with the $C_{2}(\operatorname{MAX}(H))$ and the $C_{2}[\operatorname{MIN}(H)]$ norms. If, in addition, $\xi \perp \eta$, then

$$
\begin{gathered}
\left\|\left[\begin{array}{l}
\xi \\
\eta
\end{array}\right]\right\|_{C_{2}\left(H_{r}\right)}=\left\|\left[\begin{array}{l}
\xi \\
\eta
\end{array}\right]\right\|_{C_{2}(\operatorname{MIN}(H))}=\left\|\left[\begin{array}{ll}
\xi & \eta
\end{array}\right]\right\|_{R_{2}\left(H_{c}\right)}=\max \{\|\xi\|,\|\eta\|\}, \\
\left\|\left[\begin{array}{l}
\xi \\
\eta
\end{array}\right]\right\|_{C_{2}\left[H_{r}\right]}=\left\|\left[\begin{array}{l}
\xi \\
\eta
\end{array}\right]\right\|_{C_{2}[\operatorname{MAX}(H)]}=\left\|\left[\begin{array}{ll}
\xi & \eta
\end{array}\right]\right\|_{R_{2}\left[H_{c}\right]}=\|\xi\|+\|\eta\|,
\end{gathered}
$$

$$
\left\|\left[\begin{array}{l}
\xi \\
\eta
\end{array}\right]\right\|_{C_{2}\left(H_{o}\right)}=\sqrt[4]{\|\xi\|^{4}+\|\eta\|^{4}} \text {, and }\left\|\left[\begin{array}{l}
\xi \\
\eta
\end{array}\right]\right\|_{C_{2}\left[H_{o}\right]}=\left(\|\xi\|^{\frac{4}{3}}+\|\eta\|^{\frac{4}{3}}\right)^{\frac{3}{4}}
$$

Proof. We may assume that neither $\xi$ nor $\eta$ is zero. Equations (10) and (11) are well-known, for example (10) follows from the completely isometric identifications $C_{2}\left(H_{c}\right) \cong\left(H^{2}\right)_{c} \cong C_{2}\left[H_{c}\right]$ and $R_{2}\left(H_{r}\right) \cong\left(H^{2}\right)_{r} \cong R_{2}\left[H_{r}\right]$, where $H^{2}=H \oplus H$. The first assertion after (10) follows from (10), (4), and the fact that MAX dominates the other operator space structures. Similarly the second assertion follows from (10) and (5). For Equation (12), we will use 
the completely isometric identifications $C_{2}\left[H_{r}\right] \cong \mathcal{T}\left(\bar{H}, \mathbb{C}^{2}\right)$ and $R_{2}\left[H_{c}\right] \cong$ $\mathcal{T}\left(\overline{\mathbb{C}^{2}}, H\right)$, where for Hilbert spaces $K$ and $L, \mathcal{T}(\bar{K}, L)$ denotes the family of trace-class operators from the conjugate Hilbert space of $K$ to $L$. Under the first identification, $\left[\begin{array}{l}\xi \\ \eta\end{array}\right]$ corresponds to the mapping $S: \bar{H} \rightarrow \mathbb{C}^{2}$ defined by

$$
S(\bar{\zeta})=\left[\begin{array}{l}
\langle\bar{\zeta}, \bar{\xi}\rangle \\
\langle\bar{\zeta}, \bar{\eta}\rangle
\end{array}\right]=\left[\begin{array}{l}
\langle\xi, \zeta\rangle \\
\langle\eta, \zeta\rangle
\end{array}\right] \text { for all } \zeta \in H
$$

Under the second identification, $\left[\begin{array}{ll}\xi & \eta\end{array}\right]$ corresponds to the mapping $T$ : $\overline{\mathbb{C}^{2}} \rightarrow H$ defined by

$$
S\left(\overline{\left[\begin{array}{l}
a \\
b
\end{array}\right]}\right)=\bar{a} \xi+\bar{b} \eta \text { for all } a, b \in \mathbb{C} .
$$

Routine calculations then show that

$$
\left\|\left[\begin{array}{l}
\xi \\
\eta
\end{array}\right]\right\|_{C_{2}\left[H_{r}\right]}=\|S\|_{\mathcal{T}\left(\bar{H}, \mathbb{C}^{2}\right)}=\|\xi\|+\|\eta\|
$$

and

$$
\left\|\left[\begin{array}{ll}
\xi & \eta
\end{array}\right]\right\|_{R_{2}\left[H_{c}\right]}=\|T\|_{\mathcal{T}\left(\overline{\mathbb{C}^{2}}, H\right)}=\|\xi\|+\|\eta\| .
$$

Since the norm on $C_{2}[\operatorname{MAX}(H)]$ dominates the $C_{2}\left[H_{r}\right]$ norm, it must be equal to $\|\xi\|+\|\eta\|$ too. Finally, to prove Equation (13), we compute

$$
\left\|\left[\begin{array}{l}
\xi \\
\eta
\end{array}\right]\right\|_{C_{2}\left(H_{o}\right)}=\left\|\left[\begin{array}{c}
\langle\xi, \xi\rangle \\
\langle\xi, \eta\rangle \\
\langle\eta, \xi\rangle \\
\langle\eta, \eta\rangle
\end{array}\right]\right\|^{1 / 2}=\left\|\left[\begin{array}{c}
\|\xi\|^{2} \\
0 \\
0 \\
\|\eta\|^{2}
\end{array}\right]\right\|^{1 / 2}=\sqrt[4]{\|\xi\|^{4}+\|\eta\|^{4}} .
$$

The second statement in (13) may be seen from the following argument of Pisier: It is shown in [29], Theorem 2.3 that $C_{2}\left[H_{o}\right]=H_{o} \otimes_{h} C_{2}$ is the midway interpolant between $H_{c} \otimes_{h} C_{2}$ and $H_{r} \otimes_{h} C_{2}$. But the first space may be thought of as the Schatten 2-class, and the second space as the trace class. Hence $H_{o} \otimes_{h} C_{2}$ may be identified with the Schatten $\frac{4}{3}$-class. From this the claimed statement follows easily.

Proposition 6.10. The one-sided $M$ - and L-projections for the various quantizations of $H$ are given by the following table. 


\begin{tabular}{|l|c|c|c|c|}
\hline Quantization & Left $M$-Proj's & Right $L$-Proj's & Right $M$-Proj's & Left $L$-Proj's \\
\hline$H_{c}$ & Proj $(B(H))$ & $\{0, I\}$ & $\{0, I\}$ & $\operatorname{Proj}(B(H))$ \\
\hline$H_{r}$ & $\{0, I\}$ & $\operatorname{Proj}(B(H))$ & $\operatorname{Proj}(B(H))$ & $\{0, I\}$ \\
\hline$H_{o}$ & $\{0, I\}$ & $\{0, I\}$ & $\{0, I\}$ & $\{0, I\}$ \\
\hline $\operatorname{MIN}(H)$ & $\{0, I\}$ & $\operatorname{Proj}(B(H))$ & $\{0, I\}$ & $\operatorname{Proj}(B(H))$ \\
\hline $\operatorname{MAX}(H)$ & $\operatorname{Proj}(B(H))$ & $\{0, I\}$ & $\operatorname{Proj}(B(H))$ & $\{0, I\}$ \\
\hline
\end{tabular}

where Proj $(B(H))$ is the family of orthogonal projections on $H$ and $I$ is the identity on $H$. The first three rows of the table are also valid for complete one-sided $M$ - and L-projections. For $\operatorname{MAX}(H)$ however (respectively, $\operatorname{MIN}(H)$ ), there are no nontrivial complete left- or right- $M$-projections (respectively, L-projections).

Proof. We begin by noting that a one-sided $M$ - or $L$-projection $P$ for any of the five quantizations of $H$ is necessarily an orthogonal projection on $H$ since any such $P$ is a contractive linear idempotent.

Our procedure for showing that a given entry in the table is $\{0, I\}$ is to suppose the contrary. Then there exists an orthonormal set $\{\xi, \eta\}$ with $P \xi=\xi$ and $P \eta=0$. The fact that $\|\eta+\xi\|=\sqrt{2}$ leads to a contradiction if one appeals to the appropriate formula in the previous lemma. For example, our assertion that the entries in the second and third columns of the first row are as small as possible follows immediately from this argument and (11) and (12).

The fact that the entries in the first and fourth columns of the first row are as large as possible follows almost immediately from the previous lemma. For example, we have from (10) that for any $P \in \operatorname{Proj}(B(H))$,

$$
\left\|\left[\begin{array}{c}
P \xi \\
(I d-P) \xi
\end{array}\right]\right\|_{C_{2}\left(H_{c}\right)}=\sqrt{\|P \xi\|^{2}+\|(I d-P) \xi\|^{2}}=\|\xi\|
$$

for all $\xi \in H$ and

$$
\begin{aligned}
\|P \xi+(I d-P) \eta\| & =\sqrt{\|P \xi\|^{2}+\|(I d-P) \eta\|^{2}} \\
& \leq \sqrt{\|\xi\|^{2}+\|\eta\|^{2}}=\left\|\left[\begin{array}{l}
\xi \\
\eta
\end{array}\right]\right\|_{C_{2}\left(H_{c}\right)}
\end{aligned}
$$

for all $\xi, \eta \in H$. In other words, $\nu_{P}^{c}: H_{c} \rightarrow C_{2}\left(H_{c}\right)$ is an isometry and $\mu_{P}^{c}: C_{2}\left(H_{c}\right) \rightarrow H_{c}$ is a contraction. Because of the completely isometric identification $C_{2}\left(H_{c}\right) \cong\left(H^{2}\right)_{c}$, and because for maps between Hilbert column spaces the norm coincides with the completely bounded norm, we conclude that $\nu_{P}^{c}$ and $\mu_{P}^{c}$ are in fact completely contractive. Consequently, $P$ is a complete left $M$-projection. Since $C_{2}\left(H_{c}\right) \cong C_{2}\left[H_{c}\right]$ completely isometrically, we see the other assertion. 
Thus we have completed the first row. The entries for the $H_{r}$ row follows by symmetry.

The entries in the first and third columns of the fourth row will be equal by symmetry, since $C_{2}(\mathrm{MIN}(H)) \cong R_{2}(\mathrm{MIN}(H))$ isometrically. Again arguing by contradiction and (11), we conclude that these entries are the trivial ones. Similarly by symmetry the first and third columns of the third row will be equal, and we use (13) to evaluate these. Similarly the second and fourth entries of the fourth row are equal, and we use the second statement after (10) to deduce that the listed entries are correct here. The remaining entries in the table are verified in just the same way.

Finally, we shall show that $\operatorname{MIN}(H)$ has no nontrivial complete right $L$ projections (from which the other final statements follow by duality and symmetry). To that end, assume that $P \in \operatorname{Proj}(B(H))$ is a nontrivial complete right $L$-projection for $\operatorname{MIN}(H)$. Then there exist orthonormal vectors $\xi, \eta \in H$ such that $P \xi=\xi$ and $P \eta=0$. But then using successively (11), the definition of a complete right $L$-projection, Lemma 2.2, and the "row-version" of (5), we have

$$
\begin{aligned}
& 1=\max \{\|\xi\|,\|\eta\|\} \\
& =\left\|\left[\begin{array}{ll}
\xi & \eta
\end{array}\right]\right\|_{R_{2}(\mathrm{MIN}(H))} \\
& =\left\|\left[\begin{array}{llll}
P \xi & (I d-P) \xi & P \eta & (I d-P) \eta
\end{array}\right]\right\|_{R_{2}\left(R_{2}[\operatorname{MIN}(H)]\right)}
\end{aligned}
$$

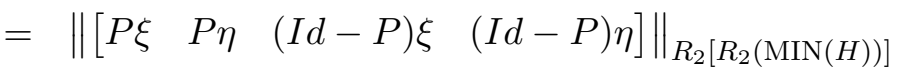

$$
\begin{aligned}
& =\left\|\left[\begin{array}{llll}
\xi & 0 & 0 & \eta
\end{array}\right]\right\|_{R_{2}\left[R_{2}(\operatorname{MIN}(H))\right]} \\
& \geq \sqrt{\left\|\left[\begin{array}{ll}
\xi & 0
\end{array}\right]\right\|_{R_{2}(\operatorname{MIN}(H))}^{2}+\left\|\left[\begin{array}{ll}
0 & \eta
\end{array}\right]\right\|_{R_{2}(\operatorname{MIN}(H))}^{2}} \\
& =\sqrt{\|\xi\|^{2}+\|\eta\|^{2}} \\
& =\sqrt{2} \text {, }
\end{aligned}
$$

a contradiction.

Using this proposition, we can identify the one-sided summands and ideals in Hilbert operator spaces. For example, the (complete) right $M$-ideals in $H_{c}$ are precisely the closed subspaces of $H$, whereas the only right $L$-summands of $H_{o}$ are $\{0\}$ and $H$.

6.11. As a final example, we note that it is proved in [13] that there exist no nontrivial complete right or left $L$-projections on a $C^{*}$-algebra. Equivalently, there exist no nontrivial complete right or left $M$-projections on the predual of a von Neumann algebra. In these results we may replace the word "complete" by "strong" (see $\S 3$ ).

Remarks added March 2001: V. Paulsen has found an elegant proof of Theorem 4.6 based on a $3 \times 3$ matrix argument. 
As we have indicated elsewhere [11], but which is appropriate to state here, Theorem 4.6 facilitates a deeper understanding of the interplay between the multiplication operation and the metric structure of an operator algebra. On the one hand, it gives more or less immediately the 'BRS' characterization of operator algebras, and the 'CES' characterization of operator modules (or more generally, the 'oplication theorem' of [10]). This was independently observed by Paulsen. On the other hand it enables one to recover the multiplication operation on a unital operator algebra from its underlying operator space structure.

To illustrate the second assertion, let us suppose that $A$ is an operator algebra with an identity of norm 1 , but that we have 'forgotten' the multiplication operation on $A$. Let us assume for a moment that we do 'remember' the identity element $e$. Form $M_{\ell}(A)$ using Theorem 4.6, and define $\theta: M_{\ell}(A) \rightarrow A$ by $\theta(T)=T(e)$. Then the product on $A$ is given by $a b=\theta\left(\theta^{-1}(a) \theta^{-1}(b)\right)$.

If we have also forgotten the specific identity element $e$, then we can only retrieve the product on $A$ up to a unitary $u$ with $u, u^{-1} \in A$. Such unitaries form a group. Indeed they are characterized by the Banach-Stone theorem for operator algebras (see e.g., the last page of [10], or [25] for the $C^{*}$-algebra case) as the elements $x_{0}$ with the property that the map $\pi: T \mapsto T\left(x_{0}\right)$ is a completely isometric surjection $M_{\ell}(A) \rightarrow A$. If $A$ is a $C^{*}$-algebra one only needs this to be an isometry, by Kadison's result [25]. We remark that from Lemma 4.5 the unitaries in a $C^{*}$-algebra correspond to linear $\varphi: A \rightarrow A$ such that $\tau_{\varphi}^{c}$ is a surjective isometry. However in this case there are other Banach space characterizations of unitaries. We are indebted to C. Akemann and N. Weaver for showing us a way to do this (see [1]). On the other hand, M. Walter has recently used an elegant $3 \times 3$ matrix trick to reconstruct the product in a unital $C^{*}$-algebra from the matrix order structure (see [37]). Given such an $x_{0}$ and $\pi$, we may again recover the product as $a b=\pi\left(\pi^{-1}(a) \pi^{-1}(b)\right)$. This is the operator algebra product on $A$ which has this unitary as the identity. This is all fairly easy to see from the Banach-Stone theorem mentioned above and basic facts about the left multiplier algebra.

Added in Proof: Around 1998, W. Werner considered left multipliers on a class of non-unital operator systems and proved an intrinsic matrix order-theoretic characterization which is analogous to our characterization of contractive left multipliers in Theorem 4.6. Indeed this insightful theorem (in an early version of [39]) provided the inspiration for our (non-order theoretic) result. He has very recently pointed out to us that one can also prove our result by using a version of the "Paulsen trick" to replace an operator space by an ordered system of the variety considered in his paper. In this context one may use a " $4 \times 4$ " matrix argument to recover our 
theorem. By this trick, the operator space multipliers in [10] and [12] may be described within Werner's framework, and some of the results from those papers may be deduced from Werner's work. Similarly the projections that Werner used in [39] are related to the one-sided complete $M$-projections of this paper.

\section{References}

[1] C. Akemann and N. Weaver, Geometric characterization of some classes of operators in $C^{*}$-algebras. Preprint, 2001.

[2] E.M. Alfsen, $M$-structure and intersection properties of balls in Banach spaces, Israel J. Math., 13 (1972), 235-245, MR 49 \#3497, Zbl 0259.46013

[3] _ Compact convex sets and boundary integrals, Erg. Math., 57, Springer Verlag, Berlin, 1971, MR 56 \#3615, Zbl 0209.42601.

[4] E.M. Alfsen and T. Andersen, Split faces of compact convex sets, Proc. London Math. Soc. (3), 21 (1970), 415-442, MR 44 \#2012, Zbl 0207.12204.

[5] E.M. Alfsen and E.G. Effros, Structure in real Banach spaces I \& II, Ann. of Math., 96 (1972), 98-173, MR 50 \#5432, Zbl 0248.46019.

[6] E.M. Alfsen and F. Schultz, On orientation and dynamics in operator algebras I, Comm. Math. Phys. (1), 194 (1998), 87-108, MR 99h:46129, Zbl 0918.46060.

[7] W.B. Arveson, Subalgebras of $C^{*}$-algebras, Acta Math., 123 (1969), 141-224, MR 40 \#6274, Zbl 0194.15701.

[8] _ Subalgebras of $C^{*}$-algebras II, Acta Math., 128 (1972), 271-308, MR 52 \#15035, Zbl 0245.46098.

[9] E. Behrends, M-structure and the Banach-Stone theorem, Lecture Notes in Math., 736, Springer-Verlag, Berlin (1979), MR 81b:46002, Zbl 0436.46013.

[10] D.P. Blecher, The Shilov boundary of an operator space and the characterization theorems, J. Funct. Anal., 182(2) (2001), 280-343, MR 2002d:46049.

[11] _ Multipliers and dual operator algebras, J. Funct. Anal., 183(2) (2001), 498525, CMP 1844216.

[12] D.P. Blecher and V.I. Paulsen, Multipliers of operator spaces and the injective envelope, Pacific J. Math., 200(1) (2001), 1-17, CMP 1863404.

[13] D. P. Blecher, R.R. Smith and V. Zarikian, Left projections on $C^{*}$-algebras, to appear.

[14] F.F. Bonsall and J. Duncan, Complete Normed Algebras, Springer-Verlag, New YorkHeidelberg, 1973, MR 54 \#11013, Zbl 0271.46039.

[15] E. Christensen, E. Effros and A. Sinclair, Completely bounded multilinear maps and $C^{*}$-algebraic cohomology, Invent. Mat., 90 (1987), 279-290, MR 89k:46084, Zbl 0646.46052.

[16] E.G. Effros, Order ideals in a $C^{*}$-algebra and its dual, Duke Math. J., 30 (1963), 391-412, MR 27 \#1847, Zbl 0117.09703.

[17] E.G. Effros, N. Ozawa and Z.J. Ruan, On injectivity and nuclearity for operator spaces, Duke Math. J., 110(3) (2001), 489-521, CMP 1869114.

[18] E.G. Effros and Z.J. Ruan, Representations of operator bimodules and their applications, J. Operator Theory, 19 (1988), 137-157, MR 91e:46077, Zbl 0705.46026. 
[19] _ On non-self-adjoint operator algebras, Proc. Amer. Math. Soc., 110 (1990), 915-922, MR 91c:47086, Zbl 0718.46020.

[20] _ Mapping spaces and liftings for operator spaces, Proc. London Math. Soc., 69 (1994), 171-197, MR 96c:46074a, Zbl 0814.47053.

[21] _ Operator Spaces, Oxford University Press, Oxford (2000), MR 2002a:46082, Zbl 0969.46002.

[22] M. Hamana, Injective envelopes of operator systems, Publ. R.I.M.S. Kyoto Univ., 15 (1979), 773-785, MR 81h:46071, Zbl 0436.46046.

[23] - Triple envelopes and Silov boundaries of operator spaces, Math. J. Toyama University, 22 (1999), 77-93, MR 2001a:46057, Zbl 0948.46045.

[24] P. Harmand, D. Werner and W. Werner, $M$-ideals in Banach spaces and Banach algebras, Lecture Notes in Mathematics, 1547, Springer-Verlag, Berlin-New York (1993), MR 94k:46022, Zbl 0789.46011.

[25] R.V. Kadison, Isometries of operator algebras, Ann. of Math. (2), 54 (1951), 325-338, MR 13,256a, Zbl 0045.06201.

[26] E. Kirchberg, On restricted perturbations in inverse images and a description of normalizer algebras in $C^{*}$-algebras, J. Funct. Anal., 129 (1995), 35-63, MR 95m:46094b, Zbl 0828.46052.

[27] E.C. Lance, Hilbert $C^{*}$-modules - A toolkit for operator algebraists, London Math. Soc. Lecture Notes, Cambridge University Press (1995), MR 96k:46100, Zbl 0822.46080.

[28] W. Paschke, Inner product modules over $B^{*}$-algebras, Trans. Amer. Math. Soc., 182 (1973), 443-468, MR 50 \#8087, Zbl 0265.46056.

[29] G. Pisier, The operator Hilbert space OH, complex interpolation and tensor norms, Memoirs Amer. Math. Soc., 585 (1996), MR 97a:46024, Zbl 0932.46046.

[30] R.T. Prosser, On the ideal structure of operator algebras, Memoirs Amer. Math. Soc., 45 (1963), MR 27 \#1846, Zbl 0125.06703.

[31] M.A. Rieffel, Morita equivalence for $C^{*}$-algebras and $W^{*}$-algebras, J. Pure Appl. Algebra, 5 (1974), 51-96, MR 51 \#3912, Zbl 0295.46099.

[32] _ Morita equivalence for operator algebras, Proceedings of Symposia in Pure Mathematics, 38 Part 1 (1982), 285-298, MR 84k:46045, Zbl 0541.46044.

[33] Z.J. Ruan, Subspaces of $C^{*}$-algebras, J. Funct. Anal., 76 (1988), 217-230, MR 89h:46082, Zbl 0646.46055.

[34] _ Injectivity of operator spaces, Trans. Amer. Math. Soc., 315 (1989), 89-104, MR 91d:46078, Zbl 0669.46029.

[35] R.R. Smith and J.D. Ward, $M$-ideal structure in Banach algebras, J. Funct. Anal., 27 (1978), 337-349, MR 80j:46087, Zbl 0369.46044.

[36] M. Tomita, Spectral theory of operator algebras, II, Math. J. Okayama Univ., 10 (1960), 19-60, Zbl 0204.14605.

[37] M. Walter, M. E. Walter, Algebraic structures determined by $3 \times 3$ matrix geometry. Preprint, 2001.

[38] K.H. Werner, A characterization of $C^{*}$-algebras by nh-projections on matrix ordered spaces. Preprint, Universitat des Saarlandes, 1978.

[39] W. Werner, Small K-groups for operator systems. Preprint, 1999. 
[40] G. Wittstock, Extensions of completely bounded module morphisms, Proceedings of conference on operator algebras and group representations, Neptum, Pitman (1983), 238-250, MR 85i:46080, Zbl 0535.46003.

[41] Matrix order and $W^{*}$-algebras in the operational approach to statistical physical systems, Comm. Math. Phys., 74 (1980), 61-70, MR 81j:81006, Zbl 0451.46046.

[42] V. Zarikian, Complete One-Sided M-Ideals in Operator Spaces, Ph.D. Thesis, UCLA.

Received December 18, 2000 and revised April 13, 2001. Blecher and Effros were partially supported by the National Science Foundation.

Department of Mathematics

UNIVERSITY OF HOUSTON

Houston, TX 77204-3008

E-mail address: dblecher@math.uh.edu

Department of Mathematics

UNIVERSITY OF CALIFORNIA

Los ANGeles, CA 90095-1555

E-mail address: ege@math.ucla.edu

Department of Mathematics

The University of Texas at Austin

Austin, TX 78712-1082

E-mail address: zarikian@math.utexas.edu 